\title{
Mono-2-ethylhexyl phthalate disrupts neurulation and modifies the embryonic redox environment and gene expression
}

\author{
Karilyn E. Sant ${ }^{\mathrm{a}}$, Dana C. Dolinoy ${ }^{\mathrm{a}, \mathrm{b}}$, Joseph L. Jilek ${ }^{\mathrm{a}}$, Maureen A. Sartor ${ }^{\mathrm{c}, \mathrm{d}}$, Craig Harris ${ }^{\mathrm{a}, \mathrm{b}}$ \\ ${ }^{a}$ Department of Environmental Health Sciences, University of Michigan School \\ of Public Health, Ann Arbor, MI 48109 \\ ${ }^{\mathrm{b}}$ Department of Nutritional Sciences, University of Michigan School of Public \\ Health, Ann Arbor, MI 48109 \\ ${ }^{\mathrm{c}}$ Department of Computational Medicine \& Bioinformatics, University of \\ Michigan Medical School, Ann Arbor, MI 48109 \\ ${ }^{\mathrm{d}}$ Department of Biostatistics, University of Michigan School of Public Health, \\ Ann Arbor, MI 48109
}

Corresponding Author: Email: charris@umich.edu

\author{
Phone: +1 (734) 936-3397 \\ Department of Environmental Health Sciences \\ University of Michigan, School of Public Health \\ M6108 SPH II
}

1415 Washington Heights, Ann Arbor MI 48109-2029 


\section{Abstract}

Mono-2-ethylhexl phthalate (MEHP) is the primary metabolite of di-2-ethylhexyl

3 phthalate (DEHP), a ubiquitous contaminant in plastics. This study sought to determine how

$4 \quad$ structural defects caused by MEHP in mouse whole embryo culture were related to temporal and

5 spatial patterns of redox state and gene expression. MEHP reduced morphology scores along

6 with increased incidence of neural tube defects. Glutathione (GSH) and cysteine (Cys)

7 concentrations fluctuated spatially and temporally in embryo (EMB) and visceral yolk sac (VYS)

8 across the $24 \mathrm{~h}$ culture. Redox potentials $\left(\mathrm{E}_{h}\right)$ for GSSG/GSH were increased by MEHP in EMB

9 (12h) but not in VYS. CySS/CyS $\mathrm{E}_{h}$ in EMB and VYS were significantly increased at $3 \mathrm{~h}$ and

$1024 \mathrm{~h}$, respectively. Gene expression at $6 \mathrm{~h}$ showed that MEHP induced selective alterations in

11 EMB and VYS for oxidative phosphorylation and energy metabolism pathways. Overall, MEHP

12 affects neurulation, alters $\mathrm{E}_{h}$, and spatially alters the expression of metabolic genes in the early 13 organogenesis-stage mouse conceptus.

15 Keywords: embryo, neural tube defects, MEHP, DEHP, visceral yolk sac, redox potential, 16 glutathione, cysteine, gene expression

\section{Introduction}

Development is a highly regulated process that relies on tightly controlled intra- and 20 intercellular signaling for normal growth. Dysregulation of embryogenesis can be caused by a 21 myriad of genetic, physiological, and environmental factors such as the chemicals found in our 22 air, water, and food. Exposure to chemical agents can alter specific signaling pathways to 
23 manipulate cell polarity and migration, membrane transport, and protein structure and function

24 [1-4]. Ultimately, these changes can manifest as spontaneous abortions, birth defects, and growth

25 deficits or predispose individuals to diseases that arise during childhood and into adulthood. One

26 such factor that can lead to this dysregulation is altered redox signaling and control, caused by

27 perturbations of the soluble thiol steady state, along with an increase in reactive oxygen species

28 (ROS) [5]. Many human and animal teratogens, compounds that produce congenital

29 malformations, are reported to act through classical oxidative stress: a change from a balanced

30 and reducing intracellular redox environment to predominantly oxidizing conditions, concurrent

31 with the generation of ROS [6]. An oxidizing environment during development has also been

32 associated with increased risk of postnatal chronic diseases including neurodegeneration,

33 hypertension, cancer, and Type II Diabetes [7-9]. Environmental contaminants, including the

34 phthalates discussed in this report, have also been shown to increase the generation of ROS [10-

35 13]. Views about the roles of increased ROS generation in biological cells and tissues have

36 changed of late because ROS are now known to have critical signaling and regulatory roles in

37 normal cell function [14-16]. These discoveries have helped dispell the previous notion that all

38 ROS are deleterious. Through the selective oxidation and reduction of protein thiols, critical cell

39 processes such as enzyme activity, receptors, transporters, transcription factors, and second

40 messenger signaling are all regulated. While related, the widespread practice of invoking

41 "oxidative stress" through the simple measurement of glutathione (GSH) depletion or increases

42 in markers of lipid peroxidation because they vary naturally and do not represent the significance

43 of the broader redox environment [17]. Many of the regulatory consequences of altered redox

44 status have been more accurately assessed by measuring intracellular redox potentials $\left(\mathrm{E}_{h}\right)$.

45 which, although based on the GSSG/GSH redox couples, are a more sensitive indicator of 
environmentally-induced perturbations to the redox steady state. Progressive increases in

47 intracellular $\mathrm{E}_{h}$ are highly correlated with changes in proliferation, differentiation, and tissue

48 patterning during embryogenesis and organogenesis [5, 18]. Altered $\mathrm{E}_{h}$ during embryonic

49 development can range from mild to severe, leading to manifestations ranging from distorted

50 signal transduction to apoptosis and necrosis, respectively. Because these consequences are

51 likely harmful, the body has an endogenous and dynamic antioxidant system to prevent and

52 protect against the more damaging consequences of oxidation. The reducing agent glutathione

53 (GSH) and related enzymes glutathione reductase, glutathione peroxidase, and glutamate-

54 cysteine ligase are primarily responsible for the maintenance of a balanced cellular $\mathrm{E}_{h}$ [17].

55 Production and recycling of these endogenous antioxidants is regulated through the induction of

56 the Nrf2 antioxidant pathway [19]. The action of other redox couples, such as reduced cysteine

57 (Cys) and oxidized cysteine (CySS) or thioredoxin, help maintain steady redox states and govern

58 redox signaling in the embryo. When the actions of all of these antioxidants and protective

enzymes are insufficient to maintain a balanced environment during development, teratogenesis can occur [5].

61
Mono-2-ethylhexyl phthalate (MEHP) is the primary metabolite of the common plasticizing agent, di-2-ethylhexyl phthalate (DEHP). DEHP has been widely incorporated into many products worldwide, but is of special concern because of its inclusion in vinyl piping and medical tubing. Evidence of phthalate transfer to the EMB and fetus has been evident through detection of MEHP in meconium, amniotic fluid, cord blood, rodent fetal tissues, and placental perfusate [20-28]. It has been previously demonstrated that MEHP can induce general oxidative stress and inflammation in various reproductive tissues and developmental tissues via the increased generation of ROS, though these studies were conducted in other model organisms and 
70 at different stages of development [12, 13, 29, 30]. It has been demonstrated that several

71 chemical compounds have the ability to modify the embryonic redox environment, and that these

72 changes can result in teratogenic outcomes [5, 31-35]. Compounds such as ethanol, diamide,

73 methylmercury, phenytoin, and L-buthionine-S,R-sulfoximine have been associated with

74 oxidation of the various tissue and fluid compartments of the conceptus, including VYS, the yolk

75 sac fluid (YSF), amniotic fluid (AF), and the EMB [34, 36-38]. These compounds are able to

76 decrease GSH concentrations and selectively increase Eh in conceptal tissues and fluids. The

77 body of evidence for other toxicants altering redox signaling in the organogenesis-stage

78 conceptus is growing, and many of these exposures are associated with adverse health outcomes

79 and structural defects.

80 Thus, it was important to determine the ontogeny of $\mathrm{E}_{h}$ across the susceptible window of

81 early organogenesis in order to identify potential windows of susceptibility for phthalate

82 exposure and relate disturbances to sensitive developmental events. The goals of this study were

83 to characterize MEHP-induced morphology and growth changes in mouse whole embryo culture

84 (mWEC), determine whether MEHP alters conceptal $\mathrm{E}_{h}$ inEMB and VYS tissues, and to identify 85 related MEHP-induced changes in gene expression.

86

\section{2. Materials \& Methods}

2.1 Chemicals and reagents

Mono-2-ethylhexyl phthalate was obtained from AccuStandard (New Haven, CT).

90 Dioctyl phthalate (DEHP), dimethyl sulfoxide (DMSO), glutathione, glutathione disulfide,

91 cysteine, cystine, $\gamma$-glutamyl-glutamate, iodoacetic acid, iodoacetamide, bicinchoninic acid,

92 RNAlater®, Tyrode's balanced salt solution, and penicillin/streptomycin (10,000 units/ml 
93 penicillin, 10,000 $\mu \mathrm{g} / \mathrm{ml}$ streptomycin sulfate) were obtained from Sigma/Aldrich (St. Louis,

94 MO). Hanks balanced salt solution (HBSS) was purchased from GIBCO/Life Technologies

95 (Grand Island, NY). Dansyl chloride was purchased from Fluka Chemie/Sigma-Aldrich (St.

96 Louis, MO). Glacial acetic acid was obtained from Fisher Scientific (Waltham, MA).

97

\subsection{Mouse whole embryo culture}

Mouse embryo culture was performed according to the procedures outlined in [39].

Briefly, female CD-1 mice were time-mated and obtained from Charles River (Portage, MI). The

101 morning of discovery of a vaginal plug was designated as gestational day (GD) 0 . Animals were maintained on a $12 \mathrm{~h}$ light-12h dark cycle and were supplied food and water ad libitum. On GD 8, female mice were euthanized with $\mathrm{CO}_{2}(10-30 \%)$ and the uterus was removed. Culture-ready conceptuses (ranging from 6-8 somites) were explanted from the uterus, removed from the 105 decidual mass, freed from the Reichert's membrane, randomized, and placed into $10 \mathrm{ml}$ culture 106 bottles containing $5 \mathrm{ml}$ of $75 \%$ heat-inactivated rat serum $/ 25 \%$ Tyrode's balanced salt solution (TBSS) and $21.5 \mu \mathrm{l}$ penicillin/streptomycin. Immediately centrifuged rat serum was collected and prepared from female Sprague Dawley rats according to approved protocols [39]. The number of conceptuses per bottle never exceeded the standard rule of 1 conceptus per $\mathrm{ml}$ of culture medium. Bottles were placed on a continuous-gassing carousel in an incubator held at $11137^{\circ} \mathrm{C}$ and supplied with $5 \% \mathrm{O}_{2} / 5 \% \mathrm{CO}_{2} / 90 \% \mathrm{~N}_{2}$. After $6 \mathrm{~h}$ in culture, the gas input was changed 112 to $20 \% \mathrm{O}_{2} / 5 \% \mathrm{CO}_{2} / 75 \% \mathrm{~N}_{2}$ to optimize growth in culture. All animal procedures were approved by the University of Michigan University Committee on Use and Care of Animals. 


\subsection{Exposure and sample collection}

DEHP and MEHP were suspended in DMSO to increase solubility in culture. DEHP and MEHP were added to the culture medium to bring the final concentration in culture to 100,250 , 500, or $1000 \mu \mathrm{g} / \mathrm{ml}(0.4-3.6 \mathrm{mM} \mathrm{MEHP}$ and 0.3-2.6 mM DEHP). These concentrations were selected based upon concentrations utilized in other phthalate whole embryo culture studies [40, 41]. WEC experiments, like cell culture experiments, frequently use concentrations greater than those observed in vivo due to the lack of direct perfusion to the tissues, and thus these concentrations are 1-3 orders of magnitude greater than those observed in human cord blood [27, 28]. DMSO was added to control bottles at a concentration of $0.05 \%(\mathrm{v} / \mathrm{v})$ in culture, equal to the volume of added DEHP and MEHP solutions. For morphology experiments, conceptuses were grown in culture for a total of $24 \mathrm{~h}$ before removal. For redox analysis, conceptuses were sampled at $0 \mathrm{~h}$ (before explant into culture), and at 1, 3, 6, 12, and $24 \mathrm{~h}$ of culture. Samples designated for RNA isolation were collected following $6 \mathrm{~h}$ in culture. At the time of removal from culture, all conceptuses were washed 2X in Hank's balanced salt solution (HBSS) and ectoplacental cones were removed. The EMB and VYS were manually separated using watchmaker's forceps. At this time, samples were either designated for morphology assessment or for redox analysis. Individual (unpooled) samples for redox analysis were placed into thiol preservation buffer (containing 5\% perchloric acid, $0.2 \mathrm{M}$ boric acid, and $10 \mu \mathrm{M} \gamma$ glutamylglutamate), snap frozen in liquid $\mathrm{N}_{2}$, and stored at $-76^{\circ} \mathrm{C}$, as specified in [18]. Tissues collected for RNA isolation were pooled ( $\mathrm{n}=5$ per sample) and placed into RNA later and stored at $-76^{0} \mathrm{C}$. 
At the conclusion of the culture period, conceptuses for morphology assessment were 140 removed from culture and washed thoroughly in HBSS without disruption. Once rinsed, 141 conceptuses were transferred into a dish containing fresh HBSS for microscopic analysis. Images 142 were taken before and after removal of the VYS on a stage micrometer-calibrated microscope, 143 and all were imaged at the same magnification. Morphological parameters were evaluated as 144 specified in [39]. In short, we evaluated quality and developmental progress of the VYS, 145 allantois, heart, flexion, caudal neural tube, forebrain, midbrain, hindbrain, otic vesicle, optic 146 cups, forelimbs, mandible, maxillary process, and counted the number of somite pairs. 147 Additionally, crown-rump length, VYS diameter, and head length were measured [39]. VYS 148 volume was calculated by applying the average radius length to the formula for spherical volume $149\left(4 / 3 \pi r^{3}\right)$. Protein quantification of tissue was done using bicinchoninic acid (BCA) assay on a 150 microplate reader [42].

\subsection{HPLC analysis of thiols and redox potentials.}

Samples were thawed on ice and prepared for HPLC analysis of soluble thiols as 154 previously described in [36, 43]. Briefly, a Waters 2695 Alliance Separations module equipped 155 with a Supelcosil LC-NH 2 column (Sigma, St. Louis, MO) was coupled with a Waters 2475 156 Fluorescence Detector. Reverse-phase chromatography was used to measure reduced glutathione 157 (GSH), oxidized glutathione disulfide (GSSG), reduced cysteine (Cys), and oxidized cystine 158 (CySS). Flow was set to $1 \mathrm{~mL} / \mathrm{min}$ using a mobile phase gradient consisting of mobile phase A 159 consisting of $80 \%$ methanol and mobile phase B consisting of $62.5 \%$ methanol, $12.5 \%$ glacial 160 acetic acid, and $214 \mathrm{mg} / \mathrm{ml}$ sodium acetate trihydrate. Peaks were visualized by fluorescence 161 detection (excitation $335 \mathrm{~nm}$, emission $518 \mathrm{~nm}$ ) and peaks were processed using the Waters 
162 Empower software (Milford, MA). The Nernst equation ( $\mathrm{pH}$ 7.4) was used for the calculation of

$163 \mathrm{E}_{h}$ for both the Cys/CySS and GSH/GSSG redox pairs: $\mathrm{E}_{h}=-264+30 \log \left([\mathrm{GSSG}] /[\mathrm{GSH}]^{2}\right)$, 164 Cys/CySS, $\mathrm{E}_{\mathrm{h}}=-250+30 \log \left([\mathrm{CySS}] /[\mathrm{Cys}]^{2}\right)$ as described previously [18]. Absolute 165 concentrations of GSH, GSSG, Cys, and CySS were calculated and normalized to tissue protein 166 concentrations determined by BCA assay.

\subsection{RNA isolation and Affymetrix microarray analysis of expression}

VYS and EMB samples collected from M100 conceptuses at the $6 \mathrm{~h}$ mark on GD8 and stored in RNAlater (Fisher Scientific) were thawed on ice and RNA was isolated following the

171 instructions of the RNeasy Mini Kit (Qiagen). The $6 \mathrm{~h}$ time point was selected because it is a 172 sensitive period of development, during which organogenesis of several major organs has begun. 173 Additionally, this time point allowed for us to assess the acute effects of MEHP exposure while 174 avoiding expression changes merely induced by the initial stress of the culture environment. 175 RNA (n=3 for each treatment, for each tissue) was submitted for Affymetrix microarray 176 processing at the University of Michigan Microarray Core Facility. Mouse MG-430 PM strip 177 arrays were processed using the IVT Express Kit (Affymetrix). RNA purity and integrity were 178 confirmed using the RNA 6000 Nano Kit for the Agilent 2100 Bioanalyzer. Raw data obtained 179 for the array was further examined for quality and supported by satisfactory scores for PM chip 180 density, RNA degradation, and standard errors. Robust multi-array average (RMA) was used for 181 background correction, normalization, and quantification of $\log _{2}$ expression using default 182 parameters in $\mathrm{R}$ v2.4.1. Principle component analysis revealed distinctions between sample 183 groups, confirming EMB and VYS tissue differences (Supplementary Figure 1). 

were examined using the Limma package in $\mathrm{R}$ 2.4.1. The ImFit function was used to downweight potential outliers, and eBayes was used to calculate p-values and FDR levels using an empirical

187 Bayesian moderated t-test and the Benjamini and Hochberg adjustment for multiple testing [44]. 188 Validation of gene expression results was done by performing quantitative PCR on selected 189 candidate genes. Enrichment of gene sets was analyzed using LRpath, and biologically and 190 statistically altered processes and pathways were identified using the GO Biological Processes 191 (http://geneontology.org/) and KEGG Pathway (http://www.genome.jp/kegg/) databases [45]. 192 Pathways containing genes with many statistical differences between control and MEHP-treated 193 tissues were considered "enriched".

\subsection{Statistical Analysis}

Values presented in this paper are means \pm standard error of the mean. Statistical outliers 197 were removed, identified as values 1.5 times the interquartile range outside of the first and third 198 quartiles of the data. One-way ANOVA with Tukey posthoc tests and independent t-tests were 199 used to assess statistical significance of differences between groups of data. A confidence level 200 of $95 \%$ was used, and p-values <0.05 were deemed as statistically significant changes.

202 3. Results

\subsection{Morphology Assessment}

After $24 \mathrm{~h}$ of culture, phthalate-treated conceptuses were examined for changes in growth and anatomical outcomes using both the parent compound (DEHP) and the metabolite (MEHP) 206 and compared using our standard morphology scoring protocol [39]. Conceptuses in mWEC 
were exposed, beginning on GD $8(0 \mathrm{~h})$, to DEHP at concentrations of $100 \mu \mathrm{g} / \mathrm{ml}$ (D100), 500 $\mu \mathrm{g} / \mathrm{ml}$ (D500), or $1000 \mu \mathrm{g} / \mathrm{ml}$ (D1000); or to MEHP at concentrations of $100 \mu \mathrm{g} / \mathrm{ml}$ (M100), 250 $\mu \mathrm{g} / \mathrm{ml}$ (M250), $500 \mu \mathrm{g} / \mathrm{ml}$ (M500), or $1000 \mu \mathrm{g} / \mathrm{ml}$ (M1000). Concentrations were selected based upon benchmark concentrations obtained from other studies [40], and were deemed to be appropriate based on the results of this study [41]. Conceptuses treated with MEHP uniformly exhibited morphological changes, while conceptuses treated with DEHP did not show any growth deficits or malformations (Fig. 1). This is consistent with the understanding that DEHP is metabolized maternally prior to conceptal exposure, and that MEHP is the active metabolite that can cause developmental defects. Conceptuses treated with MEHP were noticeably smaller than controls, and overall size decreased in a dose-dependent fashion (Fig. 2). Table 1 displays the average scores for all morphological parameters after MEHP treatment. Control conceptuses $(n=17)$ had an average total morphology score of $86.6 \pm 6.1$. None had any defects, and growth parameters were consistent with those observed in other mWEC experiments. Treatment significantly reduced many of the scores, though it seemed to have had little effect on the mandibular or forelimb parameters. Of the most commonly affected developmental outcomes, incomplete closure of the neural tube (neural tube defect) was the prominent feature. Neural tube closure was significantly delayed by MEHP treatment in the cephalic fore-, mid-, and hindbrain segments as well as in the caudal neuropore (Fig. 3).

\subsection{MEHP morphology}

The M100 group ( $\mathrm{n}=7)$ had an average total morphology score of $73.8 \pm 3.8$. Twenty nine percent of M100 embryos exhibited hypoplastic forebrain regions, and 43\% of the embryos had open neural tubes at least at one of the four neural tube regions examined (forebrain, midbrain, 
230 hindbrain, caudal neural tube). One of the seven MEHP-treated embryos developed a clear 231 blister on the prosencephalon.

The M250 (n=6) had an average total morphology score of $72.9 \pm 2.9$. All but one of the 233 embryos had an open neural tube at one or more of the neural tube regions scored. Though all of 234 the caudal neural tubes were closed in the M250 group, all structures were highly hypoplastic at 235 this locus.

The M500 (n=16) group had an average morphology score of $56.6 \pm 3.3$, indicating a 237 significant decrease in overall growth and an increase in anatomical lesions. Hypoplasia of the 238 caudal neural tube was prominent. About a third of the embryos possessed caudal neural tubes 239 that were so necrotic that they could not be fully assessed as to the status of the caudal 240 neuropore. In all of the embryos, the caudal region was truncated, hypoplastic, and presented 241 with a 'zig-zag' pattern running along the dorsal ridge. One of the embryos had complete 242 dissociation of the neural epithelium, with large blisters and severe hypoplasia in the forebrain 243 region. In approximately one-third of the M500 embryos, the pericardium was enlarged and 244 fluid-filled, and these same embryos had blood pooling in the antimesometrial end of the VYS. 245 One of the M500 embryos also had a very thin second branchial arch.

247 the conceptuses, with an average morphology score of $28.4 \pm 0.8$. Two of the seven embryos 248 examined were not viable (lacked a heartbeat) and were excluded from the morphological 249 assessment; those that survived had very weak heartbeats in terms of rate and apparent 250 contractile force. All of the M1000 embryos were hypoplastic at the caudal end, and had the 'zig251 zag' pattern running along the dorsal ridge. All lacked a posterior neuropore, had blood pooling 
252 in the antimesometrial end of the VYS, thin second branchial arches, and signs of necrosis. In

253 one of these embryos, necrotic debris was visible within the hindbrain region.

\subsection{DEHP morphology}

Compared to the MEHP-treated conceptuses, the DEHP-treated conceptuses ( $\mathrm{n}=5$ for 257 each group) were normal morphologically. All groups were morphologically similar to controls 258 without loss of viability, and therefore only gross morphological scores are shown (Figure 1). 259 Due to the lack of apparent toxicity no other detailed information on DEHP was included in this 260 report.

\subsection{Soluble Thiol Concentrations in VYS and EMB}

263 In order to illustrate the difficulties in using soluble thiol concentrations as the sole indicator of 264 redox status and the importance of using $\mathrm{E}_{h}$ as an acceptable measure of the perturbations of the 265 intracellular redox steady states, it is necessary to show how variable the oxidized and reduced 266 proportions of the GSSG/GSH and CySS/CyS are within their respective redox couples and how 267 dynamically they change both temporally and spatially over time.

3.4.1 GSH and GSSG.

270 Concentrations $(\mu \mathrm{M})$ of reduced GSH, and its oxidized counterpart, glutathione disulfide 271 (GSSG), in EMB and VYS ( Figure 4) are shown for controls, $100 \mu \mathrm{M}$ MEHP and $250 \mu \mathrm{M}$ 272 MEHP treatments sampled at 1, 3, 6, 12 and $24 \mathrm{~h}$ of mWEC culture. Qualitative comparisons 273 between EMB and VYS at any given time point show that reduced : oxidized ratios can vary 274 considerably, even across control conceptuses. In this analysis VYS is shown to maintain a 
275 greater proportion of GSH in the GSSG form, implying a greater degree of overall oxidation in

276 this tissue. Depletion of GSH and GSSG caused by MEHP treatment occurs primarily at $1 \mathrm{~h}$ and

$27712 \mathrm{~h}$ in the EMB and at $12 \mathrm{~h}$ in the VYS. In cases where a significant depletion of GSH occurs

278 due to phthalate exposure, it is of interest to note that GSSG does not usually increase as might

279 be expected. By 24h of culture, both EMB and VYS have resolved earlier fluctuations between

280 reduced and oxidized species and established a stable and reduced intracellular environment.

281

$282 \quad \underline{3.4 .2} C y S$ and $C y S S$

283 Cysteine concentrations remain relatively reduced at $1 \mathrm{~h}$ in EMB and VYS but then 284 become variably more oxidized over the $3 \mathrm{~h}$ and $6 \mathrm{~h}$ time points. In the VYS, the greatest 285 oxidation occurs at $6 \mathrm{~h}$ and with an optimal combined overall depletion at $6 \mathrm{~h}$. Similar to the 286 GSH and GSSG data shown in Figure 4, MEHP causes overall depletion without a significant 287 shift in CyS/CySS ratios. Intracellular steady state CyS concentrations are known to be regulated 288 dynamically, as CyS and CySS are shuttled between intracellular and extracellular sources [46]. 289 We were unable to measure extracellular concentrations and their dynamic flux in this analysis. 290

\subsection{Total glutathione and cysteine concentrations}

Total glutathione and cysteine concentrations are shown for the EMB and VYS (Fig. 6).

293 Total glutathione is defined as the sum of the reduced fraction concentration plus two times the 294 disulfide fraction concentration. Control total GSH concentrations peaked at $1 \mathrm{~h}$ in VYS and 295 EMB, increasing by $75 \%$ and $90 \%$ from the $0 \mathrm{~h}$ value, respectively. EMB control concentrations 296 decreased from the $1 \mathrm{~h}$ point across the remainder of the culture period, with only a slight 297 increase at $12 \mathrm{~h}$, ending at $24 \mathrm{~h}$ at concentrations slightly below the $0 \mathrm{~h}$ starting concentrations. 
298 Control patterns in the VYS were somewhat different from the EMB where concentrations 299 peaked at $1 \mathrm{~h}$ declined at $3 \mathrm{~h}$, and peaked again at $12 \mathrm{~h}$ before they also dropped below $0 \mathrm{~h}$ 300 control values at $24 \mathrm{~h}$. In MEHP conceptuses, treatment prevented the GSH concentration spike 301 at $1 \mathrm{~h}$ in the EMB (p=0.002) in both M100 and M250 concentration groups. M100 total GSH 302 concentration peaked at $3 \mathrm{~h}$, and was elevated compared to the dwindling control $(\mathrm{p}=0.073)$ and $303 \mathrm{M} 250(\mathrm{p}<0.001)$ total GSH measures. At $12 \mathrm{~h}$, total GSH in the EMB decreased in a dose304 dependent manner compared to controls (M100: $\mathrm{p}=0.035$; M250: $\mathrm{p}<0.001$ ). However, all 305 embryonic total GSH concentrations were almost identical after $24 \mathrm{~h}$ exposures. Embryonic total 306 Cys measures also followed a dose-dependent pattern over the first $6 \mathrm{~h}$ of exposure on GD8 but 307 are, however, not significant due to high variability between samples.

\subsection{Redox Potentials}

Control $\mathrm{E}_{h}$ determinations at the $24 \mathrm{~h}$ time point are concordant with values reported from similar developmental stages in other rodent in vivo and WEC studies [36, 38, 47]. After 6 h in

312 culture (GD8), EMB GSH/GSSG $\mathrm{E}_{h}$ were chemically reduced in an apparent dose-dependent 313 manner, though these observations were not statistically significant (Fig. 7). After 12 h, MEHP314 treated EMB were significantly more oxidized than control EMB, in a dose-dependent manner 315 (M100 p=0.009; M250 p<0.001). Control EMB GSH/GSSG $\mathrm{E}_{h}$ were $-176.9 \pm 1.6 \mathrm{mV}$, and the $316 \mathrm{M} 100$ and M250 groups were $-158.3 \pm 1.4$ and $-138.2 \pm 4.5 \mathrm{mV}$, respectively. After $24 \mathrm{~h}$, all 317 samples were much more chemically reduced than at previous time points, likely due to a greater 318 capacity for new GSH biosynthesis. Despite this overall reduction of $\mathrm{E}_{h}$ in both tissues, a dose319 dependent oxidation in the EMB is still apparent. In the VYS, GSH/GSSG $\mathrm{E}_{h}$ at $3 \mathrm{~h}$ were more 320 reduced with increasing doses of 100 and $250 \mu \mathrm{g} / \mathrm{ml}$ MEHP ( $\mathrm{p}=0.06$ and $\mathrm{p}=0.04$, respectively). 
321 These $3 \mathrm{~h} \mathrm{E}_{h}$ were $-145.4 \pm 4.9,-162.2 \pm 5.2,-164.5 \pm 1.1 \mathrm{mV}$ for control, M100 and M250

322 groups, respectively. After 24 h, GSH/GSSG $\mathrm{E}_{h}$ were similar across treatment groups.

In the EMB, Cys/CySS $\mathrm{E}_{h}$ was reduced by MEHP treatments after $1 \mathrm{~h}$, though only the

$324100 \mu \mathrm{g} / \mathrm{ml}$ induced a significant change ( $\mathrm{p}=0.014)$. After $3 \mathrm{~h}$, EMB were oxidized by MEHP in a

325 dose-dependent fashion though only the $250 \mu \mathrm{g} / \mathrm{ml}$ dose was statistically oxidized compared to

326 controls $(\mathrm{p}=0.009)$. EMB CyS $\mathrm{E}_{h}$ were similar for all groups at $12 \mathrm{~h}$, and only the $250 \mu \mathrm{g} / \mathrm{ml}$

327 EMB were significantly oxidized at $24 \mathrm{~h}$ compared to controls $(\mathrm{p}=0.040)$. The MEHP-treated

328 VYS Cys/CySS $E_{h}$ were reduced at the $3 \mathrm{~h}$ time point, though the $100 \mu \mathrm{g} / \mathrm{ml}$ MEHP-treated VYS

329 were most significantly reduced $(\mathrm{p}=0.014)$. At $12 \mathrm{~h}$, increasing the dose of MEHP appeared to

330 oxidize the VYS, though it was not statistically significant. At the $24 \mathrm{~h}$ time point, the controls

331 and $250 \mu \mathrm{g} / \mathrm{ml}$ MEHP-treated VYS had similar $\mathrm{E}_{h}$, but the $100 \mu \mathrm{g} / \mathrm{ml}$ MEHP-treated VYS were

332 significantly oxidized after $24 \mathrm{~h}(\mathrm{p}<0.001)$.

333

334

3.8 Microarray analysis

MEHP-induced alterations to gene expression profiles in M100 EMB and VYS were

336 examined after $6 \mathrm{~h}$ (GD8). In the VYS, 640 probes (1.4\%), corresponding to 512 genes, showed

337 an indication of differential expression $(\mathrm{p}<0.01)$ by $6 \mathrm{~h}$ MEHP treatment. Of these probes, 249

338 (38.9\%) were downregulated and 391 (61.1\%) were upregulated. In the EMB, the expression of

3391,485 probes $(3.3 \%)$, corresponding to 1,170 genes, showed an indication of differential 340 expression. Of these probes, 541 (36.4\%) were downregulated and 945 (63.6\%) were

341 upregulated. Of the genes listed above, 201 were common to both the EMB and VYS. 
KEGG pathways significantly enriched by $6 \mathrm{~h}$ MEHP treatment in the EMB and VYS are

345 shown in Table 2. "Enriched" pathways are those that contain many significantly changed genes.

346 Several similarities were noted in EMB and VYS in response to MEHP. The most significantly

347 enriched pathway in both EMB and VYS was oxidative phosphorylation $(\mathrm{p} \leq 0.001)$. Expression

348 of several NADH dehydrogenase genes was significantly decreased, including $N d u f a 3, N d u f s 5$,

$349 N d u f a 6$, and especially Ndufa7 in both EMB and VYS. In EMB, expression of several ATPases

350 and ATP synthases was also significantly altered by MEHP treatment-primarily those of

351 lysosomal and mitochondrial origin. ATPases were primarily upregulated, while ATP synthases

352 were primarily downregulated. Expression of Uqcr11, part of the ubiquinol-cytochrome c

353 reductase complex crucial for cellular respiration, was increased in the VYS but decreased in the

354 EMB.

355

The majority of enriched pathways in both tissues were, in general, metabolic pathways, 356 namely xenobiotic response and amino acid metabolic pathways. The drug metabolism 357 cytochrome P450 (CYP450) and the glutathione metabolism pathways were significantly 358 enriched in the VYS as well. Expression of the constituents of these pathways, consisting mostly 359 of CYP450 isozymes, glutathione-S-transferases, and glutathione peroxidases was significantly 360 increased. Amino acid metabolic pathways including histidine metabolism, arginine and proline 361 metabolism, and alanine, aspartate and glutamate metabolism were all enriched in both EMB 362 and VYS. Starch and sucrose metabolism was also enriched in the EMB. Retinol metabolism was enriched in the EMB, namely with regards to non-optic 364 pathways. Though involved in the metabolism of Vitamin A, many of the genes most affected by MEHP treatment were involved in the xenobiotic response and direct signal transduction during 366 embryogenesis. Retinol dehydrogenase 1 (Rdh1), the enzyme responsible for the rate-limiting 
367 step of retinaldehyde biosynthesis, was significantly decreased in the EMB following MEHP 368 treatment. As previously mentioned, several CYP450 genes were upregulated by treatment369 including CYP26 family variants. The function of the CYP26 family is necessary to convert 370 retinaldehyde into retinoic acid, and is essential for guiding posterior tissue patterning by 371 directing Hox genes for hindbrain formation. Interestingly, Hox gene expression was 372 significantly reduced for several Hoxb, Hoxc, and Hoxd variants.

373 A fraction (1.2\%) of affected genes were members of the solute carrier (SLC) gene 374 families, which are evolutionarily well-conserved across the animal kingdom [48] (Table 3). 375 Although no single family was specifically altered in the pathway analysis, the collective volume 376 of significantly altered SLC genes was notable. Though SLCs have a diverse array of functions, 377 all are involved in the transport of important cellular substrates across cellular membranes [49]. 378 The largest group of transporters significantly impacted by MEHP treatment was involved in the 379 mitochondrial transport of solutes. These mitochondrial transporters are important for 380 metabolism, namely in oxidative processes [50]. Expression of Slc7a9, involved in CySS 381 absorption and Cys/CySS redox balance, was significantly decreased in EMB [51]. Significant 382 expression changes to members of the SLC6 family, involved in neurotransmission, appear only 383 in the EMB tissue. Decreased expression was observed in several of these genes, crucial for 384 glycine, noradrenaline, and methionine homeostasis. Several patterns were observed in the 385 overall expression of these genes. Though there was a mixture or upregulated and downregulated 386 SLC family expression in the EMB, there was a strong pattern of downregulation in the VYS. 
GO Biological Processes significantly up- or down-regulated by MEHP exposure for the

390
EMB and VYS are shown in Figure 8. A p-value of less than 0.05 was used to identify significantly changed GO Biological Processes in order to enrich a large number of processes to observe directional trends in related processes. The number of processes significantly changed by MEHP treatment in the EMB, VYS, or both tissues is shown (Fig. 8A). In all, 527 GO Biological Processes were significantly impacted in the EMB, 389 were specific to the VYS, and another 167 were significantly changed in both tissues.

To identify whether the EMB and VYS tissues responded differently to MEHP treatment, the 167 Biological Processes significantly affected in both the EMB and VYS were plotted (Fig. 8B). Processes were grouped by their parent process (metabolic process, etc.) in order to visually detect patterns of altered regulation. The direction and magnitude of change in the VYS is shown on the $\mathrm{x}$-axis, and for the EMB on the y-axis. The majority of processes were downregulated in the VYS and upregulated in the EMB, and very few processes were downregulated in both tissues. Several processes, or clusters of related processes, had high magnitudes of change in both tissues, and are labeled on the plot accordingly. Vitamin transport is one of the most significantly altered processes, and is one of two processes significantly downregulated in both tissues. Two of the most significantly altered genes within this pathway were the Folrl, the primary folate receptor, and Ttpa, a transporter for Vitamin E. Oxidative phosphorylation, electron transport chain, and related processes all clustered together, significantly upregulated in the VYS and downregulated in the EMB. Another cluster of developmental processes related to kidney morphogenesis were significantly upregulated in both the EMB and VYS. Most of the processes related to cellular translation are downregulated in the VYS and upregulated in the EMB by MEHP, namely Amino acid activation. Interestingly, most processes pertaining to 
412 response to stimuli were clustered in the lower-right quadrant, correlated with the expected 413 upregulation in the VYS, but were downregulation in the EMB.

\section{4. Discussion}

Development is a tightly regulated process that is governed by integrated signaling

417 pathways and influenced by combinations of genetic and environmental cues. The environmental 418 cues come from a myriad of sources, including toxicant exposures, nutrient availability, disease, 419 maternal hormones, extracellular ligands, altered redox environments and physical stressors. The 420 specific pathways that are altered by environmental toxicants, such as MEHP, have not been well 421 characterized during mammalian organogenesis. In this study, we sought to better understand the 422 correlative spatial and temporal relationships between exposure, growth and anatomical 423 malformations, changes in redox status, and altered gene expression during embryogenesis using 424 the mWEC system.

425 By way of comparison, the stage of development studied in this report coincides with the 426 early first trimester of human gestation, during active neural tube closure but prior to the 427 establishment to active placental oxygen and nutrient exchange [52, 53]. This study design 428 allowed us to compare structural, biochemical, and regulatory consequences of MEHP exposure 429 independently in the EMB and VYS across a significant $24 \mathrm{~h}$ time span that brackets the 430 developmental period during which the embryo undergoes axial rotation, closure of the anterior 431 neural tube, initiation of an active heartbeat, vitelline circulation, and onset of forelimb 432 development [54-56]. Assessment of changes to these developmental milestones was made using 433 our standard morphology assessment [39] which is summarized in Table 1 and depicted in Figure

434 2. Results revealed an MEHP-induced decline in conceptal growth and a significantly elevated 
435 incidence of abnormally open neural tubes (NTDs) over the $24 \mathrm{~h}$ period from GD8 to GD 9. By

436 mouse GD 9, the anterior neural tube should be fully closed while the posterior neuropore

437 remains open as a pin hole while the trunk continues to elongate. The incidence of defectively 438 open neural tubes was significantly increased in culture beginning at the lowest MEHP 439 concentration tested $(100 \mu \mathrm{g} / \mathrm{ml})$. The sensitivity of CD-1 mouse conceptuses used in this study 440 was found to be much greater than rat conceptuses grown in WEC during the same relative stage 441 of development [41]. Robinson et al similarly observed abnormal neurulation in rat conceptuses, 442 but only after MEHP concentrations exceeded $600 \mu \mathrm{g} / \mathrm{ml}$ [57]. In spite of a different degree of 443 sensitivity to MEHP and obvious differences in culture conditions including: a different species

444 (Wistar rat instead of our CD-1 mouse), timing, stage of embryos at initiation of exposure (1-5 445 somites instead of our 8-10 somites), $\mathrm{O}_{2}$ gassing protocol, and duration of exposure (48 $\mathrm{h}$ instead 446 of our $24 \mathrm{~h}$ ), the overall morphological and growth outcomes were quite similar to those we 447 observed in mice. While incomplete neural tube closure was the most prominent morphological 448 defect, parameters indicative of overall growth reductions were also prominent in the treatment449 related decrease in total morphology scores (Table1, Figure 1).

450 Our conclusion that MEHP is a developmental toxicant/teratogen has also been supported 451 by several other studies which have examined effects during different phases of prenatal 452 development. Fairbairn et al observed that zebrafish embryos exposed to dibutyl phthalate during 453 embryogenesis had disrupted axial growth, resulting in severe dysmorphogenesis from 454 incomplete epiboly [58]. Janer et al investigated the consequences of MEHP-treatment in rat 455 whole embryo culture, and found that MEHP treatment in culture significantly reduced overall 456 growth [40]. Species-specific sensitivity to MEHP is not unexpected because similar differences 457 have been observed for many chemical compounds and across several species of conceptus in 
458 WEC (reviewed in [59]). Robinson et al investigated the teratogenic and gene expression 459 consequences of congruent MEHP exposures in the EMB only, and at a later time point [41]. 460 Here, we add a novel conceptal tissue comparison for gene expression, at an earlier, and 461 presumably more sensitive, time point during organogenesis.

463 the excess generation of reactive oxygen species (ROS), an identity shared with MEHP [5, 10, 464 11, 13, 60, 61]. Previous studies have demonstrated that phthalates, including MEHP, are 465 capable of inducing oxidizing conditions in the developing embryo, although sampling occurred 466 at different developmental time points and were assessed in different model systems than 467 employed here. Chu et al found increased ROS concentrations in early preimplantation embryos 468 exposed to concentrations of MEHP that were similar to those used in this study [29] as well as 469 to mono-butyl phthalate [62]. Increased ROS were also detected in phthalate-exposed zebrafish 470 eleutherolarvae [63]. The meaningful assessment of chemically-altered redox states in relation to actual effects

472 on biological outcomes is difficult to make due to the inability to account for subtle spatial and 473 temporal changes in redox states, as well as the lack of a defining quantitative endpoint for 474 evaluation of redox-related toxicity. In this study we have chosen to use the estimation of 475 intracellular redox potentials $\left(\mathrm{E}_{h}\right)$ as a readout for chemically-induced perturbation of the redox 476 steady state in conceptuses [47]. Maintenance of a proper redox environment has been shown to 477 be crucial for the developmental determination of cell fates. Oxidation of specific protein thiol 478 residues, act as sensitive "on/off” switches for regulating numerous signaling pathways that are 479 influenced by disruption of the redox steady state, often irrespective of changes in the magnitude 480 of total GSH or total CyS concentrations. The consequences of increasing (oxidizing) $\mathrm{E}_{h}$ values 
481 have been extensively studied of late and are known to progressively direct cell fate towards 482 proliferation, differentiation, apoptosis, and, eventually, necrosis [61, 64]. Typically, the more 483 reducing environments are correlated with cells undergoing proliferation, while more oxidative 484 environments are associated with differentiation or ultimately, cell death. For related reasons, the 485 protein thiol oxidation elicited as a result of increased ROS production and the other factors that 486 disturb $\mathrm{E}_{h}$ have also been implicated in controlling the pluripotency of stem cells, which can 487 occur in the formation of cancers [65-68], and which has been implicated in mechanisms leading 488 to anatomical birth defects $[5,61,69]$. Based on a growing body of evidence an $\mathrm{E}_{h}$ shift of +20 489 $\mathrm{mV}$ (GSH/GSSG redox couple) is sufficient to alter cell activity, moving from proliferation to 490 differentiation.; A $50 \mathrm{mV}$ increase creates conditions that can lead to death [70]. At the $12 \mathrm{~h}$ time 491 point in WEC, conceptuses exposed to 100 or $250 \mu \mathrm{g} / \mathrm{ml} \mathrm{MEHP} \mathrm{resulted} \mathrm{in} \mathrm{Eh} \mathrm{increases} \mathrm{of}$ 492 approximately 20 and $40 \mathrm{mV}$, respectively, in the EMB (Figure 7), which is a sufficient $\mathrm{E}_{h}$ 493 change to alter patterns of proliferation and differentiation in the EMB. The specific redox494 sensitive pathways, signaling nodes, and target proteins that may be responsible for preventing 495 timely neural tube closure have not yet been identified.

Numerous temporal and spatial changes in the proportion of oxidized (GSSG, CySS) and 497 reduced (GSH, Cys) species in the VYS and EMB occur across the $24 \mathrm{hr}$ time course under 498 normal culture conditions. Total GSH and CyS concentrations (reduced $+2 \mathrm{X}$ oxidized thiol), as 499 well as the relative proportion of oxidized and reduced species they represent, fluctuate naturally 500 in controls and may not be directly correlated with any specific growth or differentiation event. 501 The addition of MEHP produces a generally consistent, but fluctuating, pattern of total GSH and 502 CyS reduction without eliciting a significant net proportional shift towards greater oxidation (as 503 indicated by $\mathrm{E}_{h}$ ). The initial spike in total GSH and total CyS observed at $1 \mathrm{~h}$ is very transient 
504 and is believed to result as an adaptation from the initial shock of explant and introduction into 505 culture, athough, this effect was modified by MEHP. These increases and their subsequent return 506 to the normal range are not accompanied by a net shift in $\mathrm{E}_{h}$ (Figure 7). Across the $24 \mathrm{~h}$ time 507 course, the $\mathrm{E}_{h}$ values remain relatively stable in the VYS until the tissues become highly reduced 508 at $24 \mathrm{~h}$. This pattern also holds for the EMB with the exception of the $12 \mathrm{~h}$ time point where a 509 significant dose-dependent oxidation occurs. Again, this time-specific oxidation at $12 \mathrm{~h}$ 510 coincides with several critical physiological, differentiation, and growth-sensitive developmental 511 transitions such as neural tube closure, which might be related mechanistically. The control 512 mouse VYS and EMB also appear to be much more oxidized at the onset of culture on GD8 513 compared to the rat (GD10) at a comparable stage of development. This trend continues through $51412 \mathrm{~h}$ of culture in the mouse. This may indicate that the enzymes responsible for GSH 515 biosynthesis and regulation are late to mature in the mouse, as has been shown for other 516 metabolic and biotransformation enzyme activities [71]. Optimal reducing environments, in 517 which $\mathrm{GSH}_{h}$ values drop below $-230 \mathrm{mV}$, are reached and sustained by $24 \mathrm{~h}$ in $\mathrm{mWEC}$ (GD 9).

The meaningful identification and comparison of disparate measures such as growth, 519 morphogenesis, and redox status would be greatly enhanced with the identification and 520 characterization of precise quantitative endpoints for embryotoxicity. In order to narrow the 521 search to relevant targets and pathways we have attempted to superimpose an analysis of gene 522 expression over the growth, anatomical, and biochemical parameters. Because a complete 523 temporal ontogeny profile was not possible for the current analysis we chose to evaluate only the 524 spatial VYS and EMB expressions at the $6 \mathrm{~h}$ time point. The rationale for assessment of 525 expression at $6 \mathrm{~h}$ was based on a perceived need to avoid the period of culture adjustments $(1 \mathrm{~h}$ $5263 \mathrm{~h}$ ) but still to preceed the major genetic and physiological events that accompany the onset of 
527 active heartbeat and related metabolic events around the $12 \mathrm{~h}$ point. The $6 \mathrm{~h}$ time point

528 sepresents a cumulative expression profile representative of all normal and MEHP-induced

529 events elicited over the first $6 \mathrm{~h}$ of culture. One major consideration that is often overlooked in

530 the evaluation of expression data in complex tissues such as are seen in the developing conceptus

531 is the contribution of multiple cell types to the overall expression profile. This is particularly

532 important in the conceptus where many different types of cells are constantly emerging as the

533 three primary germ layers differentiate, migrate, expand, and die as they follow the

534 developmental program. The VYS is most often discarded as irrelevant in developmental

535 assessments although its active circulation, metabolic contributions, and nutritive functions are

536 intimately associated and completely essential to EMB growth and development. Comparative

537 gene expression data (Table 2) shows five MEHP-disrupted pathways that were identified from

538 the KEGG database and which are common to both EMB and VYS. These can be collectively

539 identified as pathways related to oxidative phosphorylation and amino acid metabolism. It is not

540 surprising, perhaps, that the EMB also has altered expression in pathways associated with

541 nutrient metabolism, protein recycling and membrane transport, while the VYS displays

542 alterations in pathways required for xenobiotic biotransformation such as glutathione

543 metabolism, drug metabolism-cytochrome P450, and selenocompound metabolism. The VYS

544 also shows significant changes in pathways related to ribosome biogenesis and aminoacyl-tRNA

545 biosynthesis, which have been noted in other studies as being consequences of amino acid

546 starvation and the likely activation or misregulation of autophagy $[36,72,73]$. The perturbation

547 of expression related to the latter pathways are in accord with other studies using a variety of

548 xenobiotics and chemical modulators which suggest that the disruption of conceptal nutrient

549 uptake may be a contributory factor in these outcomes $[36,72,73]$. This concept agrees with 
550 previous studies showing that inhibition of the histiotrophic nutrition pathways (HNP), through

551 which the vast majority of conceptal nutrients are obtained, results in convincing proteomic

552 evidence that the activation of ribosome biogenesis and autophagy pathways are selective

553 indicators of toxicity. We have previously demonstrated that MEHP significantly reduces HNP

554 activity in the mouse model used in the study [74]. This indicates that MEHP-induced changes in

555 nutrient availability and processing could be a factor in observed developmental toxicity.

In this study, mRNA expression related to numerous pathways involved in amino acid

557 metabolism were disrupted, such as the metabolism of alanine, aspartate, glutamate, arginine,

558 proline, and histidine. Additional indirect amino acid metabolic pathways, such as GSH

559 metabolism and TCA cycle were also affected. Because all of these pathways rely on the supply

560 of amino acids, it is possible that MEHP treatment is inducing a form of amino acid starvation

561 through decreased nutrient availability as previously found [74]. Amino acid starvation has been

562 discovered to have numerous physiological and metabolic consequences, namely effects on

563 energy metabolism including oxidative phosphorylation $[75,76]$. MEHP has been demonstrated

564 to decrease protein uptake into the conceptus, reducing the amino acids available for protein

565 synthesis and metabolism [74]. It is therefore likely that MEHP-induced amino acid starvation

566 could have numerous downstream consequences affecting signaling and overall growth and

567 differentiation. The decreased size of embryos exposed to MEHP raises questions regarding the

568 implications of phthalates on both growth and also potential metabolic diseases later in life.

569 Furthermore, vitamin transport was downregulated in both the EMB and VYS, supporting the 570 concept that a nutritional deficiency could be occurring. Transport of folate, one of the primary

571 nutrients protective against NTDs, was downregulated, and provides a potential mechanism for 572 investigation for MEHP-related NTDs. 
574 and VYS due to phthalate treatment based on the bioinformatics analysis, but was upregulated in

575 the VYS and downregulated in the EMB. The changes in oxidative phosphorylation processes

576 observed are indicative of MEHP-induced dysregulation of energy metabolism processes.

577 Increases in the expression of ATPases, some of the most redox-sensitive proteins in the cell,

578 suggest the necessity to maintain cellular homeostasis and potential mitochondrial ion gradients

579 during toxicant-induced physiological changes in the cell [77-82]. Likewise, decreases in the

580 expression of ATP synthases in the EMB could be indicative of decreased substrates available

581 for energy production (ATP-form) in the cell. This suggests that the ability to produce ATP is

582 compromised, and yet the energy demands are growing.

583 The brain has very high energy demands, and relies heavily on oxidative phosphorylation

584 and mitochondrial function [83]. It is not surprising that this time in development would be very

585 susceptible to changes in oxidative phosphorylation, since large increases in mitochondrial 586 density and mitochondrial proteins are initiated at the beginning of neurogenesis [84]. Changes

587 to the oxidative phosphorylation pathway have been previously demonstrated to be altered by 588 exposures in the brain and NTDs $[85,86]$. Because oxidative phosphorylation requires the 589 coordination of numerous enzymes and energy substrates, as well as maintenance of a high590 energy proton gradient, these processes are often decreased in starved cells. These cells then rely 591 on the more efficient but lower energy payout process of glycolysis. During this phase of 592 organogenesis, oxidative phosphorylation processes are only initiating, and the embryo mostly 593 relies on glycolysis for ATP generation. However, inhibition of oxidative phosphorylation during 594 this window frequently results in NTDs and embryolethality in mouse models [54-56]. In this 595 study, the GO Biological Process oxidative phosphorylation was decreased in the EMB, though 
596 further mechanistic work is necessary to determine whether this decrease contributes to the NTD 597 phenotypes observed in this study.

598 Disruption of oxidative phosphorylation and amino acid metabolism, oxidation of GSH/GSSG 599 redox potential, decreased vitamin transport processes, compounded with prior evidence of 600 decreased nutritional clearance, suggest that MEHP treatment can produce severe nutritional 601 deficiencies during early organogenesis [74]. Often during such deficiencies, processes 602 governing mitochondrial function are stimulated or supported at the expense of cytosolic 603 processes [87]. Expression changes to enzymes involved with amino acid metabolism, GSH 604 metabolism, TCA cycle, and oxidative phosphorylation, coupled to the expression changes 605 observed in the SLC25 mitochondrial transporters class suggest that this shunting and 606 prioritization of the maintenance of mitochondrial functions is beginning to occur at this $6 \mathrm{~h}$ time 607 point. Metabolic biological processes significantly changed by MEHP exposure were regulated 608 differently in the EMB and VYS, often with increased metabolic capacity in the VYS and 609 decreased activity in the EMB. It is likely that the VYS is preserving its function as a metabolic 610 barrier for the EMB, but this is occurring at the energetic expense of the EMB. Further 611 mechanistic work to study the metabolic consequences of MEHP exposure on EMB and VYS 612 mitochondrial function, is necessary to characterize the specific energetic effects in the EMB and 613 VYS.

Although the KEGG data shown in Table 2 gives a good picture of the major expression 615 changes induced by MEHP in VYS and EMB, they do not show many important additional 616 details about the direction of expression changes which are instructive for optimal interpretation.

617 In order to extend this analysis, we have plotted expression data sorted by gene ontology (GO) 618 coding. Altered gene expression patterns in MEHP-exposed conceptal tissues are shown in 
Figure 8 as a distribution of GO Biological Processes. The GO terms represent molecular events or other beginning-to-end operations that are integrated across the spectrum of biological organization from cells to the whole organism. Expression changes for EMB are distributed along the $\mathrm{Y}$-axis where positive values represent increased expression and negative values decreased expression. The X-axis shows distribution in the VYS from positive to negative in like fashion. General patterns of distribution show that the prominent effect of MEHP exposure in the whole conceptus is to increase gene expression in the EMB and decrease expression in the VYS. GO terms differentially clustered in this manner are those associated with amino acid metabolism, metabolic processes, cellular processes, and localization, indicating that the EMB is mounting a positive adaptive response to the chemical insult. Concurrent down-regulation of the same processes in the VYS suggests that it is undergoing a more conservative adaptive response. GO terms most prominent in the quadrant where gene expression is increased in both EMB and VYS are those for developmental processes, biological regulation, negative regulation of peptidyl-serine phosphorylation, and a cluster related to kidney development. An even smaller number of GO terms mapped to the quadrant for increased VYS expression and decreased EMB expression where clusters for oxidative phosphorylation \& etc. and iron-sulfur cluster assembly along with response to stimulus, metabolic processes and localization predominate. The only GO term that was found to be associated with decreased expression in both EMB and VYS mapped to vitamin transport. This analysis suggests that the two major common alterations in MEHPinduced gene expression: oxidative phosphorylation and amino acid metabolism/activation (Table 2) identified by KEGG pathways, represent increased VYS expression/decreased EMB expression and increased EMB expression/decreased VYS expression, respectively. Decreased oxidative phosphorylation and increased amino acid metabolism are conditions generally 
642 associated with the activation of autophagy, otherwise known as 'programmed cell survival'.

643 This pattern agrees with proteomic results from previous studies where rat conceptuses were

644 exposed to GSH modulators, protease inhibitors, and embryotoxins where changes in protein

645 concentrations mapped to similar pathways $[36,72,73]$.

Results from this spatial and temporal evaluation of EMB and VYS morphology, redox

647 status, and gene expression during early mouse organogenesis, highlights several of the inherent

648 difficulties in identifying specific mechanistic associations between changes in redox status and

649 adverse structural and functional outcomes. The phthalate, MEHP, elicits significant increases in

$650 \mathrm{E}_{h}$ in both EMB and VYS which are based on the altered relative concentrations of oxidized and

651 reduced thiol species for the GSSG/GSH and CySS/CyS redox couples. Neither thiol

652 concentrations nor their respective $\mathrm{E}_{h}$ values remain constant during mouse organogenesis, but

653 rather, fluctuate spatially and temporally in EMB and VYS. The lack of concordance between

654 the two tissues and over time suggest that, even though EMB and VYS are intimately connected

655 and share a common circulatory system, they are regulated independently. The observed

656 differential patterns of gene expression support this conclusion and suggest possible MEHP

657 targets to examine for their roles in eliciting embryotoxicity and causing neural tube defects.

658 Gene expression pathways related to oxidative phosphorylation were significantly upregulated in

659 the VYS and downregulated in the EMB, while pathways for amino acid metabolism were

660 significantly upregulated in the EMB and downregulated in the VYS, suggesting attempts to

661 differentially increase energy production or metabolism in one tissue and to conserve activity in

662 another. The roles of these changes, along with decreases in processes such as vitamin transport,

663 other solute carrier transporters, developmental processes, and metabolic processes, have yet to

664 be linked specifically and mechanistically with redox states or the specific etiology of neural 
665 tube defects. Based on the results presented here, it is not clear whether MEHP induces changes 666 in $E_{h}$ that lead to altered differential gene expression and improper neural tube closure or 667 whether MEHP first alters gene expression that then leads to alterations in $\mathrm{E}_{h}$ and the formation 668 of neural tube defects.

669

\section{5. Acknowledgements}

671 We would like to acknowledge Grace Kuan, Lindsey Jacobs, and Erin Scarlett for their

672 laboratory support. This work was supported by the Bill and Melinda Gates Foundation (Grand

673 Challenges Explorations - Rd. 7) and the University of Michigan National Institute of 674 Environmental Health Sciences (NIEHS) Core Center "Lifestage Environmental Exposures and 675 Disease" (M-LEEaD; P30 ES017885). Support was also provided to K.E.S. by an NRSA 676 institutional training grant (T32 ES007062). 


\section{Figure Captions}

Figure 1. MEHP treatment reduces overall morphological score. Unlike the parent compound DEHP, metabolite MEHP is able to reduce overall morphological scores in a dose-dependent manner after $24 \mathrm{~h}$ treatment in WEC. N=5 for all DEHP groups; sample sizes for MEHP groups are presented in Table 1. * indicates a significant change $(\mathrm{p}<0.05)$ in the MEHP group from the control at this dose. $* * *$ indicates a very significant change $(\mathrm{p}<0.001)$ in the MEHP group from the control at this dose. No significant changes were observed in DEHP-treated conceptuses.

Figure 2. MEHP decreases embryonic size and induces malformations. Images taken of the exposed conceptuses exposed to MEHP beginning on GD8 and cultured for $24 \mathrm{~h}$ reveal a gradual decrease in overall size and an increase in malformations as media MEHP concentrations increase. All images were

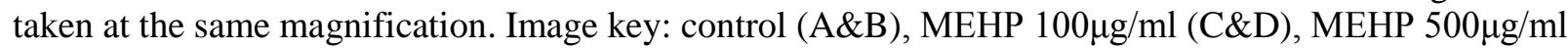
(E\&F), MEHP 1mg/ml (G\&H).

Figure 3. MEHP treatment impairs neural tube closure in WEC. An unusually high number of open neural tubes were observed in the MEHP-treated groups after $24 \mathrm{~h}$ treatment. Some of those malformations are shown above. Overall, with increased concentration of MEHP in the culture media, there is a reduction of neural tube scores. The highest possible total neural tube score attainable is 20, and scores are reduced by characteristics such as an open neural tube, necrotic debris present in the neural tube, and hypoplastic tissues around the brain regions. $* * *$ indicates a very significant change $(\mathrm{p}<0.001)$ in the MEHP group from the control at this dose.

Figure 4. Ontogeny of reduced and oxidized glutathione measurements in EMB and VYS following MEHP treatment. Black fractions represent the concentrations $(\mu \mathrm{M})$ of reduced glutathione $(\mathrm{GSH})$, and gray fractions represent the concentrations $(\mu \mathrm{M})$ of oxidized glutathione (GSSG). Results from each analysis group represent a total of 4-8 EMB or VYS samples, collected from individual conceptuses and randomized from multiple litters.

Figure 5. Ontogeny of reduced and oxidized cysteine measurements in EMB and VYS following MEHP treatment. Black fractions represent the concentrations $(\mu \mathrm{M})$ of reduced cysteine $(\mathrm{Cys})$, and gray fractions represent the concentrations $(\mu \mathrm{M})$ of oxidized cystine (CySS). Results from each analysis group represent a total of 4-8 EMB or VYS samples, collected from individual conceptuses and randomized from multiple litters.

Figure 6. Ontogeny of total thiol measurements in EMB and VYS following MEHP treatment on GD 89. Total concentrations are equal to the sum of the reduced and $2 x$ the oxidized fractions of the thiol, since disulfides were measured. Top left: total glutathione concentrations measured in EM; top right: total glutathione concentrations measured in VYS; Bottom left: total cysteine concentrations measured in EMB; and bottom right: total cysteine concentrations measured in VYS.

Figure 7. Ontogenies of redox profiles in conceptal tissues following treatment MEHP treatment on GD8-9. Embryonic redox profiles for glutathione (top left) and cysteine (bottom left) redox couples are shown, as well as VYS redox profiles for glutathione (top right) and cysteine (bottom right) redox couples.

Figure 8. GO Biological Processes significantly affected by MEHP treatment in both the EMB and VYS. (A) The number of GO Biological Processes significantly altered by MEHP treatment $(\mathrm{p}<0.05)$ in the EMB, VYS, and both tissues. (B) A plot of direction and magnitude of change (log fold change) of GO Biological Processes significantly affected by MEHP treatment in both the EMB and VYS. Direction and magnitude of process change in the VYS is shown on the $\mathrm{x}$-axis, and for the EMB on the y-axis. 
725 Processes, or clusters of related processes, which had high degrees of change due to MEHP treatment in 726 both the EMB and VYS are labeled on the graph.

727

728

729

730

731 
Table 1. MEHP treatment significantly alters developmental progress in WEC. Though DEHP treatment produced little change in most measurable developmental parameters, the metabolite MEHP significantly altered many defect scores. Values presented are the means \pm standard error of the mean. $* \mathrm{p}<0.05$, $* * \mathrm{p}<0.01, * * * \mathrm{p}<0.001,---$ indicates that the specimens were too necrotic to accurately assess this parameter.

\begin{tabular}{|l|c|c|c|c|c|}
\hline & Control & $\begin{array}{c}\text { MEHP } \\
(100 \mu \mathrm{g} / \mathrm{ml})\end{array}$ & $\begin{array}{c}\text { MEHP } \\
(250 \mu \mathrm{g} / \mathrm{ml})\end{array}$ & $\begin{array}{c}\text { MEHP } \\
(500 \mu \mathrm{g} / \mathrm{ml})\end{array}$ & $\begin{array}{c}\text { MEHP } \\
(1 \mathrm{mg} / \mathrm{ml})\end{array}$ \\
\hline$n$ & 17 & 7 & 6 & 16 & 5 \\
\hline Yolk Sac & $5.0 \pm 0$ & $4.6 \pm 0.3$ & $5.0 \pm 0$ & $2.1 \pm 0.3$ & $0.4 \pm 0.2$ \\
\hline Allantois & $3.0 \pm 0$ & $2.6 \pm 0.2$ & $3.0 \pm 0$ & $1.8 \pm 0.2$ & $1.2 \pm 0.4$ \\
\hline Flexion & $4.7 \pm 0.1$ & $3.9 \pm 0.4$ & $4.3 \pm 0.2$ & $4.0 \pm 0.1$ & $4.0 \pm 0$ \\
\hline Heart & $3.8 \pm 0.1$ & $3.7 \pm 0.2$ & $3.5 \pm 0.2$ & $3.3 \pm 0.1$ & $2.6 \pm 0.2$ \\
\hline Caudal Neural Tube & $4.2 \pm 0.1$ & $3.7 \pm 0.2$ & $4.0 \pm 0$ & $1.3 \pm 0.5$ & -- \\
\hline Hindbrain & $4.9 \pm 0.1$ & $3.6 \pm 0.4$ & $2.8 \pm 0.3$ & $3.0 \pm 0.3$ & $2.0 \pm 0$ \\
\hline Midbrain & $4.9 \pm 0.1$ & $3.6 \pm 0.5$ & $2.8 \pm 0.4$ & $2.6 \pm 0.3$ & $2.0 \pm 0$ \\
\hline Forebrain & $4.6 \pm 0.2$ & $3.3 \pm 0.5$ & $2.8 \pm 0.5$ & $2.9 \pm 0.2$ & $2.2 \pm 0.2$ \\
\hline Otic Vesicle & $4.5 \pm 0.2$ & $3.3 \pm 0.3$ & $2.5 \pm 0.2$ & $2.7 \pm 0.3$ & $2.4 \pm 0.2$ \\
\hline Optic Cup & $4.6 \pm 0.2$ & $3.7 \pm 0.4$ & $2.8 \pm 0.5$ & $1.9 \pm 0.3$ & $1.0 \pm 0$ \\
\hline Branchial & $2.7 \pm 0.1$ & $2.6 \pm 0.2$ & $2.7 \pm 0.2$ & $2.0 \pm 0.1$ & $2.0 \pm 0$ \\
\hline Maxillary & $3.0 \pm 0$ & $1.9 \pm 0.1$ & $2.0 \pm 0$ & $1.7 \pm 0.1$ & $1.0 \pm 0$ \\
\hline Mandible & $2.0 \pm 0$ & $2.0 \pm 0.2$ & $2.0 \pm 0$ & $1.9 \pm 0.1$ & $2.0 \pm 0$ \\
\hline Forelimbs & $2.1 \pm 0.2$ & $1.9 \pm 0.3$ & $2.2 \pm 0.2$ & $2.0 \pm 0.2$ & $1.6 \pm 0.2$ \\
\hline & & & & & \\
\hline Somites $(\#$ pairs) & $24.6 \pm 0.4$ & $21.0 \pm 0.8$ & $23.7 \pm 0.8$ & $18.8 \pm 1.5$ & --- \\
\hline VYS volume $\left(\mu 1^{3}\right)$ & $17.9 \pm 1.4$ & $13.8 \pm 1.4$ & $17.1 \pm 0.8$ & $13.8 \pm 1.1$ & $10.2 \pm 0.9$ \\
\hline $\begin{array}{l}\text { Crown-Rump Length } \\
\text { (mm) }\end{array}$ & $2.9 \pm 0.1$ & $2.7 \pm 0.1$ & $2.9 \pm 0.1$ & $2.5 \pm 0.1$ & $1.7 \pm 0.2$ \\
\hline Head Length $(m m)$ & $1.5 \pm 0$ & $1.2 \pm 0.1$ & $1.4 \pm 0.1$ & $1.2 \pm 0$ & $1.0 \pm 0.1$ \\
\hline TOTAL SCORE & $86.6 \pm 1.5$ & $73.8 \pm 3.8$ & $72.9 \pm 2.9$ & $56.6 \pm 3.3$ & $28.4 \pm 0.8$ \\
\hline
\end{tabular}

\begin{tabular}{|l|l|}
\hline $\mathbf{P}>0.05$ & \\
\hline $\mathbf{P}<0.05$ & \\
\hline $\mathbf{P}<0.01$ & \\
\hline $\mathbf{P}<0.001$ & \\
\hline
\end{tabular}


742 Table 2. EMB and VYS pathways with significantly altered gene expression by $6 \mathrm{~h}$ MEHP treatment in 743 WEC. Significantly enriched KEGG pathways in the EMB (shown left) and the VYS (shown right) 744 include many metabolic pathways, including severaldependent upon the redox environment, and 745 pathways associated with the nervous system.

\begin{tabular}{|c|c|c|c|c|c|}
\hline Pathways significantly enriched in EMB tissue & Odds Ratio & P-value & Pathways significantly enriched in VYS tissue & Odds Ratio & P-value \\
\hline Oxidative phosphorylation & 4.136 & 0.001 & Oxidative phosphorylation & 8.109 & 0.000 \\
\hline Alanine, aspartate and glutamate metabolism & 8.517 & 0.002 & Parkinson's disease & 7.795 & 0.000 \\
\hline Retinol metabolism & 5.637 & 0.005 & Glutathione metabolism & 13.522 & 0.000 \\
\hline Phagosome & 3.060 & 0.008 & Alzheimer's disease & 5.166 & 0.000 \\
\hline Proteasome & 5.231 & 0.010 & Aminoacyl-tRNA biosynthesis & 11.307 & 0.000 \\
\hline Rheumatoid arthritis & 3.816 & 0.011 & Histidine metabolism & 11.235 & 0.002 \\
\hline Collecting duct acid secretion & 6.880 & 0.012 & N-Glycan biosynthesis & 8.051 & 0.002 \\
\hline Purine metabolism & 2.679 & 0.014 & Antigen processing and presentation & 7.024 & 0.003 \\
\hline Arginine and proline metabolism & 4.135 & 0.023 & Drug metabolism - cytochrome P450 & 6.644 & 0.004 \\
\hline Vasopressin-regulated water reabsorption & 4.610 & 0.024 & Selenocompound metabolism & 14.129 & 0.006 \\
\hline Starch and sucrose metabolism & 4.713 & 0.028 & Huntington's disease & 3.321 & 0.007 \\
\hline Histidine metabolism & 5.117 & 0.040 & Alanine, aspartate and glutamate metabolism & 8.852 & 0.007 \\
\hline \multirow[t]{3}{*}{ Parkinson's disease } & 2.633 & 0.047 & Arginine and proline metabolism & 5.335 & 0.015 \\
\hline & & & Ribosome biogenesis in eukaryotes & 4.276 & 0.022 \\
\hline & & & Complement and coagulation cascades & 3.972 & 0.039 \\
\hline
\end{tabular}


Table 3. Solute carrier (SLC) family gene expression is significantly impacted by $6 \mathrm{~h}$ MEHP treatment in WEC. The mitochondrial transport family (SLC25) represents the most significantly altered family of transporters, crucial for the catabolic processes of the cell.

\begin{tabular}{|c|c|c|c|c|}
\hline & & & nes sig & anged \\
\hline SLC family & Function & EMB & VYS & TOTAL \\
\hline 1 & glial high affinity glutamate/neutral amino acid transporter & 1 & 2 & 3 \\
\hline 4 & bicarbonate transporters & 6 & 2 & 8 \\
\hline 5 & inositol/sodium-dependent glucose transporters & 2 & 1 & 3 \\
\hline 6 & neurotransmitter transporters & 3 & 0 & 3 \\
\hline 7 & cationic/L-type amino acid transporters & 3 & 2 & 5 \\
\hline 8 & sodium/calcium exchangers & 1 & 1 & 2 \\
\hline 9 & sodium/hydrogen exchangers & 3 & 3 & 6 \\
\hline 10 & sodium/bile salt exchangers & 0 & 1 & 1 \\
\hline 11 & proton-coupled divalent metal ion transporters & 1 & 0 & 1 \\
\hline 12 & electroneutral cation-coupled chloride cotransporters & 2 & 1 & 3 \\
\hline 13 & sodium sulphate/carboxylate exchangers & 0 & 1 & 1 \\
\hline 16 & monocarboxylic acid transporters & 6 & 2 & 8 \\
\hline 17 & phosphate/organic anion transporters & 2 & 1 & 3 \\
\hline 19 & folate/thiamine transporters & 1 & 1 & 2 \\
\hline 21 & organic anion transporters & 4 & 3 & 7 \\
\hline 22 & organic cation/anion transporters & 4 & 0 & 4 \\
\hline 23 & ascorbate/nucleobase transporters & 1 & 1 & 2 \\
\hline 24 & sodium/potassium/calcium exchangers & 1 & 0 & 1 \\
\hline 25 & mitochondrial transporters & 9 & 6 & 15 \\
\hline 26 & sulfate/anion transporters & 2 & 2 & 4 \\
\hline 29 & nucleoside transporters & 1 & 0 & 1 \\
\hline 30 & zinc transporters & 2 & 2 & 4 \\
\hline 31 & copper transporters & 1 & 0 & 1 \\
\hline 33 & acetyl-CoA transporters & 0 & 1 & 1 \\
\hline 35 & nucleotide sugar transporters & 8 & 1 & 9 \\
\hline 36 & proton-coupled amino acid transporters & 0 & 1 & 1 \\
\hline 37 & sugar phosphate exchangers & 1 & 0 & 1 \\
\hline 38 & sodium-amino acid cotransporters & 1 & 0 & 1 \\
\hline 39 & zinc/metal ion transporters & 1 & 3 & 4 \\
\hline 44 & choline transporter-like family & 1 & 2 & 3 \\
\hline 45 & putative sugar transporters & 2 & 1 & 3 \\
\hline 46 & folate transporters & 1 & 1 & 2 \\
\hline
\end{tabular}




\section{References}

753 [1] Cai J, Cheng A, Luo Y, Lu C, Mattson MP, Rao MS, et al. Membrane properties of rat embryonic multipotent neural stem cells. J Neurochem. 2004;88:212-26.

[2] Cove DJ. The generation and modification of cell polarity. Journal of Experimental Botany. 2000;51:831-8.

[3] Thiery JP, Duband JL, Tucker GC. Cell Migration in the Vertebrate Embryo: Role of Cell Adhesion and Tissue Environment in Pattern Formation. Annual Review of Cell Biology. 1985;1:91-113.

[4] Tsujioka M. Cell migration in multicellular environments. Development, growth \& differentiation. 2011;53:528-37.

[5] Hansen JM, Harris C. Redox control of teratogenesis. Reproductive Toxicology. 2013;35:165-79.

[6] Jones DP. Redefining oxidative stress. Antioxidants \& redox signaling. 2006;8:1865-79.

[7] Ornoy A. Prenatal origin of obesity and their complications: Gestational diabetes, maternal overweight and the paradoxical effects of fetal growth restriction and macrosomia. Reproductive Toxicology. 2011;32:205-12.

[8] Wan J, Winn LM. In utero-initiated cancer: The role of reactive oxygen species. Birth Defects Research Part C: Embryo Today: Reviews. 2006;78:326-32.

[9] Jeng W, Wong AW, Ting-A-Kee R, Wells PG. Methamphetamine-enhanced embryonic oxidative DNA damage and neurodevelopmental deficits. Free Radical Biology and Medicine. 2005;39:317-26.

[10] Zhou L, Beattie MC, Lin C-Y, Liu J, Traore K, Papadopoulos V, et al. Oxidative Stress and PhthalateInduced Down-Regulation of Steroidogenesis in MA-10 Leydig Cells*. Reproductive toxicology (Elmsford, NY). 2013;0:10.1016/j.reprotox.2013.07.025.

[11] Rusyn I, Kadiiska MB, Dikalova A, Kono H, Yin M, Tsuchiya K, et al. Phthalates rapidly increase production of reactive oxygen species in vivo: role of Kupffer cells. Molecular pharmacology. 2001;59:744-50.

[12] Erkekoğlu P, Rachidi W, Yüzügüllü OG, Giray B, Öztürk M, Favier A, et al. Induction of ROS, p53, p21 in DEHP- and MEHP-exposed LNCaP cells-protection by selenium compounds. Food and Chemical Toxicology. 2011;49:1565-71.

[13] Tetz LM, Cheng AA, Korte CS, Giese RW, Wang P, Harris C, et al. Mono-2-ethylhexyl phthalate induces oxidative stress responses in human placental cells in vitro. Toxicology and Applied Pharmacology. 2013;268:47-54.

[14] Ray PD, Huang B-W, Tsuji Y. Reactive oxygen species (ROS) homeostasis and redox regulation in cellular signaling. Cellular signalling. 2012;24:981-90.

[15] D'Autreaux B, Toledano MB. ROS as signalling molecules: mechanisms that generate specificity in ROS homeostasis. Nat Rev Mol Cell Biol. 2007;8:813-24.

[16] Finkel T. Signal transduction by reactive oxygen species. The Journal of cell biology. 2011;194:7-15.

[17] Schafer FQ, Buettner GR. Redox environment of the cell as viewed through the redox state of the glutathione disulfide/glutathione couple. Free Radical Biology and Medicine. 2001;30:1191-212.

[18] Harris C, Hansen J. Oxidative Stress, Thiols, and Redox Profiles. In: Harris C, Hansen JM, editors. Developmental Toxicology: Humana Press; 2012. p. 325-46.

[19] Surh YJ, Kundu JK, Na HK. Nrf2 as a master redox switch in turning on the cellular signaling involved in the induction of cytoprotective genes by some chemopreventive phytochemicals. Planta medica. 2008;74:1526-39.

[20] Erkekoglu P, Rachidi W, Yuzugullu OG, Giray B, Favier A, Ozturk M, et al. Evaluation of cytotoxicity and oxidative DNA damaging effects of di(2-ethylhexyl)-phthalate (DEHP) and mono(2-ethylhexyl)phthalate (MEHP) on MA-10 Leydig cells and protection by selenium. Toxicology and Applied Pharmacology. 2010;248:52-62. 
[21] Lin S, Ku H-Y, Su P-H, Chen J-W, Huang P-C, Angerer J, et al. Phthalate exposure in pregnant women and their children in central Taiwan. Chemosphere. 2011;82:947-55.

[22] Lin L. [Levels of environmental endocrine disruptors in umbilical cord blood and maternal blood of low-birth-weight infants]. Zhōnghuá yùfáng-yīxué zázhì. 2008;42:177-80.

[23] Huang P-C, Kuo P-L, Chou Y-Y, Lin S-J, Lee C-C. Association between prenatal exposure to phthalates and the health of newborns. Environment International. 2009;35:14-20.

[24] Mose T, Mortensen GK, Hedegaard M, Knudsen LE. Phthalate monoesters in perfusate from a dual placenta perfusion system, the placenta tissue and umbilical cord blood. Reproductive Toxicology. 2007;23:83-91.

[25] Stroheker T, Regnier J-F, Lassurguere J, Chagnon M-C. Effect of in utero exposure to di-(2ethylhexyl)phthalate: Distribution in the rat fetus and testosterone production by rat fetal testis in culture. Food and Chemical Toxicology. 2006;44:2064-9.

[26] Calafat AM, Brock JW, Silva MJ, Gray Jr LE, Reidy JA, Barr DB, et al. Urinary and amniotic fluid levels of phthalate monoesters in rats after the oral administration of di(2-ethylhexyl) phthalate and di-n-butyl phthalate. Toxicology. 2006;217:22-30.

[27] Latini G, Felice Cd, Presta G, Vecchio Ad, Paris I, Ruggieri F, et al. In Utero Exposure to Di-(2Ethylhexyl)phthalate and Duration of Human Pregnancy. Environmental health perspectives. 2003;111:1783-5.

[28] Latini G, De Felice C, Presta G, Del Vecchio A, Paris I, Ruggieri F, et al. Exposure to Di(2ethylhexyl)phthalate in Humans during Pregnancy. Neonatology. 2003;83:22-4.

[29] Chu D-P, Tian S, Qi L, Hao C-J, Xia H-F, Ma X. Abnormality of maternal-to-embryonic transition contributes to MEHP-induced mouse 2-cell block. Journal of Cellular Physiology. 2013;228:753-63.

[30] Onorato TM, Brown PW, Morris PL. Mono-(2-ethylhexyl) Phthalate Increases Spermatocyte Mitochondrial Peroxiredoxin 3 and Cyclooxygenase 2. Journal of Andrology. 2008;29:293-303.

[31] Hansen JM, Gong S-G, Philbert M, Harris C. Misregulation of gene expression in the redox-sensitive NF-kb-dependent limb outgrowth pathway by thalidomide. Developmental Dynamics. 2002;225:186-94.

[32] Hansen JM, Carney EW, Harris C. Altered differentiation in rat and rabbit limb bud micromass cultures by glutathione modulating agents. Free Radical Biology and Medicine. 2001;31:1582-92.

[33] McNutt TL, Harris C. Lindane embryotoxicity and differential alteration of cysteine and glutathione levels in rat embryos and visceral yolk sacs. Reproductive Toxicology. 1994;8:351-62.

[34] Hiranruengchok R, Harris C. Glutathione Oxidation and Embryotoxicity Elicited by Diamide in the Developing Rat Conceptus in Vitro. Toxicology and Applied Pharmacology. 1993;120:62-71.

[35] Sahambi SK, Hales BF. Exposure to 5-Bromo-2'-deoxyuridine induces oxidative stress and activator protein-1 DNA binding activity in the embryo. Birth Defects Research Part A: Clinical and Molecular Teratology. 2006;76:580-91.

[36] Harris C, Shuster DZ, Roman Gomez R, Sant KE, Reed MS, Pohl J, et al. Inhibition of glutathione biosynthesis alters compartmental redox status and the thiol proteome in organogenesis-stage rat conceptuses. Free Radical Biology and Medicine. 2013;63:325-37.

[37] Thompson SA, White CC, Krejsa CM, Eaton DL, Kavanagh TJ. Modulation of Glutathione and Glutamate-L-cysteine Ligase by Methylmercury during Mouse Development. Toxicological Sciences. 2000;57:141-6.

[38] Jilek JL, Sant KE, Cho KH, Reed MS, Pohl J, Hansen JM, et al. Ethanol Attenuates Histiotrophic Nutrition Pathways and Alters the Intracellular Redox Environment and Thiol Proteome during Rat Organogenesis. Toxicological Sciences. 2015.

[39] Harris C. Rodent Whole Embryo Culture. In: Harris C, Hansen JM, editors. Developmental Toxicology: Humana Press; 2012. p. 215-37. 
[40] Janer G, Verhoef A, Gilsing HD, Piersma AH. Use of the rat postimplantation embryo culture to assess the embryotoxic potency within a chemical category and to identify toxic metabolites. Toxicology in Vitro. 2008;22:1797-805.

[41] Robinson JF, Verhoef A, van Beelen VA, Pennings JLA, Piersma AH. Dose-response analysis of phthalate effects on gene expression in rat whole embryo culture. Toxicology and Applied Pharmacology. 2012;264:32-41.

[42] Stoscheck C. Quantification of Protein. Methods in Enzymology. 1990;182:50-69.

[43] Jones DP, Carlson JL, Samiec PS, Sternberg Jr P, Mody Jr VC, Reed RL, et al. Glutathione measurement in human plasma: Evaluation of sample collection, storage and derivatization conditions for analysis of dansyl derivatives by HPLC. Clinica Chimica Acta. 1998;275:175-84.

[44] Smyth GK. Linear models and empirical bayes methods for assessing differential expression in microarray experiments. Statistical applications in genetics and molecular biology. 2004;3:Article3.

[45] Sartor MA, Leikauf GD, Medvedovic M. LRpath: a logistic regression approach for identifying enriched biological groups in gene expression data. Bioinformatics. 2009;25:211-7.

[46] Banerjee R. Redox outside the Box: Linking Extracellular Redox Remodeling with Intracellular Redox Metabolism. Journal of biological chemistry. 2012;287:4397-402.

[47] Hansen JM, Harris C. Glutathione during embryonic development. Biochimica et Biophysica Acta (BBA) - General Subjects. 2015;1850:1527-42.

[48] Hoglund PJ, Nordstrom KJ, Schioth HB, Fredriksson R. The solute carrier families have a remarkably long evolutionary history with the majority of the human families present before divergence of Bilaterian species. Molecular biology and evolution. 2011;28:1531-41.

[49] Institute of Biochemistry and Molecular Medicine. SLC Tables. Bioparadigmsorg,. Bern, Switzerland University of Bern; 2004.

[50] Palmieri F. The mitochondrial transporter family SLC25: Identification, properties and physiopathology. Molecular Aspects of Medicine. 2013;34:465-84.

[51] National Center for Biotechnology Information. SLC7A9. Gene Database2014.

[52] Jauniaux E, Watson AL, Hempstock J, Bao YP, Skepper JN, Burton GJ. Onset of maternal arterial blood flow and placental oxidative stress. A possible factor in human early pregnancy failure. The American journal of pathology. 2000;157:2111-22.

[53] Jauniaux E, Watson A, Burton G. Evaluation of respiratory gases and acid-base gradients in human fetal fluids and uteroplacental tissue between 7 and 16 weeks' gestation. American Journal of Obstetrics and Gynecology. 2001;184:998-1003.

[54] Li K, Li Y, Shelton JM, Richardson JA, Spencer E, Chen ZJ, et al. Cytochrome c Deficiency Causes Embryonic Lethality and Attenuates Stress-Induced Apoptosis. Cell. 2000;101:389-99.

[55] Nonn L, Williams RR, Erickson RP, Powis G. The Absence of Mitochondrial Thioredoxin 2 Causes Massive Apoptosis, Exencephaly, and Early Embryonic Lethality in Homozygous Mice. Molecular and cellular biology. 2003;23:916-22.

[56] Piruat JI, Pintado CO, Ortega-Sáenz P, Roche M, López-Barneo J. The Mitochondrial SDHD Gene Is Required for Early Embryogenesis, and Its Partial Deficiency Results in Persistent Carotid Body Glomus Cell Activation with Full Responsiveness to Hypoxia. Molecular and cellular biology. 2004;24:10933-40.

[57] Robinson JF, van Beelen VA, Verhoef A, Renkens MFJ, Luijten M, van Herwijnen MHM, et al. Embryotoxicant-Specific Transcriptomic Responses in Rat Postimplantation Whole-Embryo Culture. Toxicological Sciences. 2010;118:675-85.

[58] Fairbairn EA, Bonthius J, Cherr GN. Polycyclic aromatic hydrocarbons and dibutyl phthalate disrupt dorsal-ventral axis determination via the $\mathrm{Wnt} / \mathrm{\beta}$-catenin signaling pathway in zebrafish embryos. Aquatic Toxicology. 2012;124-125:188-96. 
[59] Webster WS, Brown-Woodman PD, Ritchie HE. A review of the contribution of whole embryo culture to the determination of hazard and risk in teratogenicity testing. The International journal of developmental biology. 1997;41:329-35.

[60] Wang W, Craig ZR, Basavarajappa MS, Hafner KS, Flaws JA. Mono-(2-ethylhexyl) phthalate induces oxidative stress and inhibits growth of mouse ovarian antral follicles. Biol Reprod. 2012;87:152.

[61] Hansen JM. Oxidative stress as a mechanism of teratogenesis. Birth defects research Part C, Embryo today : reviews. 2006;78:293-307.

[62] Chu D-P, Tian S, Sun D-G, Hao C-J, Xia H-F, Ma X. Exposure to mono-n-butyl phthalate disrupts the development of preimplantation embryos. Reproduction, Fertility and Development. 2013;25:1174-84.

[63] Xu H, Shao X, Zhang Z, Zou Y, Wu X, Yang L. Oxidative stress and immune related gene expression following exposure to di-n-butyl phthalate and diethyl phthalate in zebrafish embryos. Ecotoxicology and environmental safety. 2013;93:39-44.

[64] Aw TY. Cellular Redox: A Modulator of Intestinal Epithelial Cell Proliferation2003.

[65] Droge W. Free radicals in the physiological control of cell function. Physiological reviews. 2002;82:47-95.

[66] Shi X, Zhang Y, Zheng J, Pan J. Reactive oxygen species in cancer stem cells. Antioxidants \& redox signaling. 2012;16:1215-28.

[67] Pervaiz S, Taneja R, Ghaffari S. Oxidative stress regulation of stem and progenitor cells. Antioxidants \& redox signaling. 2009;11:2777-89.

[68] Acharya A, Das I, Chandhok D, Saha T. Redox regulation in cancer: a double-edged sword with therapeutic potential. Oxidative medicine and cellular longevity. 2010;3:23-34.

[69] Kovacic P, Somanathan R. Mechanism of teratogenesis: electron transfer, reactive oxygen species, and antioxidants. Birth defects research Part C, Embryo today : reviews. 2006;78:308-25.

[70] Harris C, Hansen JM. Oxidative stress, thiols, and redox profiles. Methods Mol Biol. 2012;889:32546.

[71] Harris C, Wang S-W, Lauchu JJ, Hansen JM. Methanol metabolism and embryotoxicity in rat and mouse conceptuses: comparisons of alcohol dehydrogenase (ADH1), formaldehyde dehydrogenase (ADH3), and catalase. Reproductive Toxicology. 2003;17:349-57.

[72] Harris C, Jilek JL, Sant KE, Pohl J, Reed M, Hansen JM. Amino acid starvation induced by protease inhibition produces differential alterations in redox status and the thiol proteome in organogenesisstage rat embryos and visceral yolk sacs. Journal of nutritional biochemistry. 2015;26:1589-98.

[73] Jilek JL, Sant KE, Cho KH, Reed MS, Pohl J, Hansen JM, et al. Ethanol Attenuates Histiotrophic Nutrition Pathways and Alters the Intracellular Redox Environment and Thiol Proteome during Rat Organogenesis. Toxicological Sciences. 2015;147:475-89.

[74] Sant KE, Dolinoy DC, Jilek JL, Shay BJ, Harris C. Mono-2-ethylhexyl phthalate (MEHP) alters histiotrophic nutrition pathways and epigenetic processes in the developing conceptus. Journal of nutritional biochemistry. 2016;27:211-8.

[75] Johnson MA, Vidoni S, Durigon R, Pearce SF, Rorbach J, He J, et al. Amino Acid Starvation Has Opposite Effects on Mitochondrial and Cytosolic Protein Synthesis. PLoS ONE. 2014;9:e93597.

[76] Harris C, Jilek JL, Sant KE, Pohl J, Reed MS, Hansen JM. Amino Acid Starvation Induced by Protease Inhibition Produces Differential Alterations in Redox Status and the Thiol Proteome in OrganogenesisStage Rat Embryos and Visceral Yolk Sacs. Journal of nutritional biochemistry. 2015.

[77] Petrushanko IY, Yakushev S, Mitkevich VA, Kamanina YV, Ziganshin RH, Meng X, et al. Sglutathionylation of the Na,K-ATPase catalytic alpha subunit is a determinant of the enzyme redox sensitivity. J Biol Chem. 2012;287:32195-205.

[78] Liu J, Kennedy DJ, Yan Y, Shapiro Jl. Reactive Oxygen Species Modulation of Na/K-ATPase Regulates Fibrosis and Renal Proximal Tubular Sodium Handling. Int J Nephrol. 2012;2012:381320. 
[79] Petrushanko I, Bogdanov N, Bulygina E, Grenacher B, Leinsoo T, Boldyrev A, et al. Na-K-ATPase in rat cerebellar granule cells is redox sensitive. American journal of physiology Regulatory, integrative and comparative physiology. 2006;290:R916-25.

[80] Comellas AP, Dada LA, Lecuona E, Pesce LM, Chandel NS, Quesada N, et al. Hypoxia-Mediated Degradation of $\mathrm{Na}$,K-ATPase via Mitochondrial Reactive Oxygen Species and the Ubiquitin-Conjugating System. Circulation Research. 2006;98:1314-22.

[81] Liu J, Tian J, Haas M, Shapiro JI, Askari A, Xie Z. Ouabain interaction with cardiac Na+/K+-ATPase initiates signal cascades independent of changes in intracellular $\mathrm{Na}+$ and $\mathrm{Ca} 2+$ concentrations. J Biol Chem. 2000;275:27838-44.

[82] Formentini L, Sanchez-Arago M, Sanchez-Cenizo L, Cuezva JM. The mitochondrial ATPase inhibitory factor 1 triggers a ROS-mediated retrograde prosurvival and proliferative response. Mol Cell. 2012;45:731-42.

[83] Kann O, Kovacs R. Mitochondria and neuronal activity. American journal of physiology Cell physiology. 2007;292:C641-57.

[84] Cordeau-Lossouarn L, Vayssiere JL, Larcher JC, Gros F, Croizat B. Mitochondrial maturation during neuronal differentiation in vivo and in vitro. Biology of the cell / under the auspices of the European Cell Biology Organization. 1991;71:57-65.

[85] Hong Y, Piao F, Zhao Y, Li S, Wang Y, Liu P. Subchronic exposure to arsenic decreased Sdha expression in the brain of mice. Neurotoxicology. 2009;30:538-43.

[86] Wlodarczyk BJ, Cabrera RM, Hill DS, Bozinov D, Zhu H, Finnell RH. Arsenic-induced gene expression changes in the neural tube of folate transport defective mouse embryos. Neurotoxicology. 2006;27:54757.

[87] Lin TC, Chen YR, Kensicki E, Li AY, Kong M, Li Y, et al. Autophagy: resetting glutamine-dependent metabolism and oxygen consumption. Autophagy. 2012;8:1477-93. 
Table 1. MEHP treatment significantly alters developmental progress in WEC. Though DEHP treatment produced little change in most measurable developmental parameters, the metabolite MEHP significantly altered many defect scores. Values presented are the means \pm standard error of the mean. ${ }^{*} \mathrm{p}<0.05$, $* * \mathrm{p}<0.01, * * * \mathrm{p}<0.001,---$ indicates that the specimens were too necrotic to accurately assess this parameter.

\begin{tabular}{|l|c|c|c|c|c|}
\hline & Control & $\begin{array}{c}\text { MEHP } \\
(100 \mu \mathrm{g} / \mathrm{ml})\end{array}$ & $\begin{array}{c}\text { MEHP } \\
(250 \mu \mathrm{g} / \mathrm{ml})\end{array}$ & $\begin{array}{c}\text { MEHP } \\
(500 \mu \mathrm{g} / \mathrm{ml})\end{array}$ & $\begin{array}{c}\text { MEHP } \\
(1 \mathrm{mg} / \mathrm{ml})\end{array}$ \\
\hline$n$ & 17 & 7 & 6 & 16 & 5 \\
\hline Yolk Sac & $5.0 \pm 0$ & $4.6 \pm 0.3$ & $5.0 \pm 0$ & $2.1 \pm 0.3$ & $0.4 \pm 0.2$ \\
\hline Allantois & $3.0 \pm 0$ & $2.6 \pm 0.2$ & $3.0 \pm 0$ & $1.8 \pm 0.2$ & $1.2 \pm 0.4$ \\
\hline Flexion & $4.7 \pm 0.1$ & $3.9 \pm 0.4$ & $4.3 \pm 0.2$ & $4.0 \pm 0.1$ & $4.0 \pm 0$ \\
\hline Heart & $3.8 \pm 0.1$ & $3.7 \pm 0.2$ & $3.5 \pm 0.2$ & $3.3 \pm 0.1$ & $2.6 \pm 0.2$ \\
\hline Caudal Neural Tube & $4.2 \pm 0.1$ & $3.7 \pm 0.2$ & $4.0 \pm 0$ & $1.3 \pm 0.5$ & -- \\
\hline Hindbrain & $4.9 \pm 0.1$ & $3.6 \pm 0.4$ & $2.8 \pm 0.3$ & $3.0 \pm 0.3$ & $2.0 \pm 0$ \\
\hline Midbrain & $4.9 \pm 0.1$ & $3.6 \pm 0.5$ & $2.8 \pm 0.4$ & $2.6 \pm 0.3$ & $2.0 \pm 0$ \\
\hline Forebrain & $4.6 \pm 0.2$ & $3.3 \pm 0.5$ & $2.8 \pm 0.5$ & $2.9 \pm 0.2$ & $2.2 \pm 0.2$ \\
\hline Otic Vesicle & $4.5 \pm 0.2$ & $3.3 \pm 0.3$ & $2.5 \pm 0.2$ & $2.7 \pm 0.3$ & $2.4 \pm 0.2$ \\
\hline Optic Cup & $4.6 \pm 0.2$ & $3.7 \pm 0.4$ & $2.8 \pm 0.5$ & $1.9 \pm 0.3$ & $1.0 \pm 0$ \\
\hline Branchial & $2.7 \pm 0.1$ & $2.6 \pm 0.2$ & $2.7 \pm 0.2$ & $2.0 \pm 0.1$ & $2.0 \pm 0$ \\
\hline Maxillary & $3.0 \pm 0$ & $1.9 \pm 0.1$ & $2.0 \pm 0$ & $1.7 \pm 0.1$ & $1.0 \pm 0$ \\
\hline Mandible & $2.0 \pm 0$ & $2.0 \pm 0.2$ & $2.0 \pm 0$ & $1.9 \pm 0.1$ & $2.0 \pm 0$ \\
\hline Forelimbs & $2.1 \pm 0.2$ & $1.9 \pm 0.3$ & $2.2 \pm 0.2$ & $2.0 \pm 0.2$ & $1.6 \pm 0.2$ \\
\hline & & & & & \\
\hline Somites (\# pairs) & $24.6 \pm 0.4$ & $21.0 \pm 0.8$ & $23.7 \pm 0.8$ & $18.8 \pm 1.5$ & -- \\
\hline VYS volume $\left(\mu l^{3}\right)$ & $17.9 \pm 1.4$ & $13.8 \pm 1.4$ & $17.1 \pm 0.8$ & $13.8 \pm 1.1$ & $10.2 \pm 0.9$ \\
\hline $\begin{array}{l}\text { Crown-Rump Length } \\
\text { (mm) }\end{array}$ & $2.9 \pm 0.1$ & $2.7 \pm 0.1$ & $2.9 \pm 0.1$ & $2.5 \pm 0.1$ & $1.7 \pm 0.2$ \\
\hline Head Length $(\mathrm{mm})$ & $1.5 \pm 0$ & $1.2 \pm 0.1$ & $1.4 \pm 0.1$ & $1.2 \pm 0$ & $1.0 \pm 0.1$ \\
\hline TOTAL SCORE & $86.6 \pm 1.5$ & $73.8 \pm 3.8$ & $72.9 \pm 2.9$ & $56.6 \pm 3.3$ & $28.4 \pm 0.8$ \\
\hline
\end{tabular}

\begin{tabular}{|l|l|}
\hline $\mathbf{P}>0.05$ & \\
\hline $\mathbf{P}<0.05$ & \\
\hline $\mathbf{P}<0.01$ & \\
\hline $\mathbf{P}<0.001$ & \\
\hline
\end{tabular}


Table 2. EMB pathways with significantly altered gene expression by $6 \mathrm{~h}$ MEHP treatment in WEC. Significantly enriched KEGG pathways (shown left) include many metabolic pathways and those dependent upon the redox environment. Significantly depleted pathways (shown right) include pathways involved in signal transduction, including several crucial for neurodevelopment.

\begin{tabular}{|c|c|c|c|c|c|}
\hline Pathways significantly enriched in EMB tissue & Odds Ratio & P-value & Pathways significantly depleted in EMB tissue & Odds Ratio & P-value \\
\hline Oxidative phosphorylation & 4.136 & 0.001 & Fc gamma R-mediated phagocytosis & 0.032 & 0.001 \\
\hline Alanine, aspartate and glutamate metabolism & 8.517 & 0.002 & Ribosome & 0.022 & 0.003 \\
\hline Retinol metabolism & 5.637 & 0.005 & Pathways in cancer & 0.322 & 0.005 \\
\hline Phagosome & 3.060 & 0.008 & Intestinal immune network for $\operatorname{Ig} \mathrm{A}$ production & 0.008 & 0.007 \\
\hline Proteasome & 5.231 & 0.010 & Calcium signaling pathway & 0.217 & 0.009 \\
\hline Rheumatoid arthritis & 3.816 & 0.011 & Wnt signaling pathway & 0.215 & 0.013 \\
\hline Collecting duct acid secretion & 6.880 & 0.012 & Axon guidance & 0.203 & 0.017 \\
\hline Purine metabolism & 2.679 & 0.014 & Prostate cancer & 0.140 & 0.021 \\
\hline Arginine and proline metabolism & 4.135 & 0.023 & Amoebiasis & 0.173 & 0.024 \\
\hline Vasopressin-regulated water reabsorption & 4.610 & 0.024 & Pancreatic cancer & 0.118 & 0.028 \\
\hline Starch and sucrose metabolism & 4.713 & 0.028 & Pyruvate metabolism & 0.042 & 0.032 \\
\hline Histidine metabolism & 5.117 & 0.040 & $\mathrm{ABC}$ transporters & 0.045 & 0.032 \\
\hline \multirow[t]{3}{*}{ Parkinson's disease } & 2.633 & 0.047 & Adherens junction & 0.155 & 0.042 \\
\hline & & & $\begin{array}{l}\text { Arrhythmogenic right ventricular cardiomyopathy } \\
\text { (ARVC) }\end{array}$ & 0.158 & 0.043 \\
\hline & & & $\mathrm{T}$ cell receptor signaling pathway & 0.245 & 0.047 \\
\hline
\end{tabular}


Table 3. VYS pathways with significantly altered gene expression by $6 \mathrm{~h}$ MEHP treatment in WEC. Significantly enriched KEGG pathways (shown left) are similar to EMB, including pathways central to metabolism, and adaptation of redox environment. Significantly depleted pathways (shown right) are also similar for both tissues, including pathways involved in signal transduction, including several crucial for neurodevelopment.

\begin{tabular}{|c|c|c|c|c|c|}
\hline Pathways significantly enriched in VYS tissue & $\begin{array}{l}\text { Odds } \\
\text { Ratio }\end{array}$ & P-value & $\begin{array}{l}\text { Pathways significantly depleted in VYS } \\
\text { tissue }\end{array}$ & $\begin{array}{l}\text { Odds } \\
\text { Ratio }\end{array}$ & $\begin{array}{l}\mathrm{P} \text { - } \\
\text { value }\end{array}$ \\
\hline Oxidative phosphorylation & 8.109 & 0.000 & Neuroactive ligand-receptor interaction & 0.127 & 0.000 \\
\hline Parkinson's disease & 7.795 & 0.000 & Tight junction & 0.059 & 0.001 \\
\hline Glutathione metabolism & 13.522 & 0.000 & Axon guidance & 0.108 & 0.005 \\
\hline Alzheimer's disease & 5.166 & 0.000 & Fc gamma R-mediated phagocytosis & 0.068 & 0.007 \\
\hline Aminoacyl-tRNA biosynthesis & 11.307 & 0.000 & Leukocyte transendothelial migration & 0.093 & 0.007 \\
\hline Histidine metabolism & 11.235 & 0.002 & Cell adhesion molecules (CAMs) & 0.105 & 0.007 \\
\hline N-Glycan biosynthesis & 8.051 & 0.002 & Long-term depression & 0.037 & 0.007 \\
\hline Antigen processing and presentation & 7.024 & 0.003 & Hypertrophic cardiomyopathy (HCM) & 0.057 & 0.008 \\
\hline Drug metabolism - cytochrome P450 & 6.644 & 0.004 & $\begin{array}{l}\text { Arrhythmogenic right ventricular } \\
\text { cardiomyopathy (ARVC) }\end{array}$ & 0.049 & 0.008 \\
\hline Selenocompound metabolism & 14.129 & 0.006 & Wnt signaling pathway & 0.158 & 0.009 \\
\hline Huntington's disease & 3.321 & 0.007 & Jak-STAT signaling pathway & 0.160 & 0.014 \\
\hline Alanine, aspartate and glutamate metabolism & 8.852 & 0.007 & Dilated cardiomyopathy & 0.089 & 0.015 \\
\hline Arginine and proline metabolism & 5.335 & 0.015 & Pathways in cancer & 0.345 & 0.016 \\
\hline Ribosome biogenesis in eukaryotes & 4.276 & 0.022 & Osteoclast differentiation & 0.145 & 0.017 \\
\hline \multirow[t]{9}{*}{ Complement and coagulation cascades } & 3.972 & 0.039 & ECM-receptor interaction & 0.095 & 0.019 \\
\hline & & & ErbB signaling pathway & 0.105 & 0.020 \\
\hline & & & Hepatitis C & 0.176 & 0.022 \\
\hline & & & $\mathrm{T}$ cell receptor signaling pathway & 0.151 & 0.023 \\
\hline & & & Regulation of actin cytoskeleton & 0.293 & 0.028 \\
\hline & & & Amoebiasis & 0.153 & 0.030 \\
\hline & & & Fc epsilon RI signaling pathway & 0.117 & 0.034 \\
\hline & & & Glioma & 0.088 & 0.038 \\
\hline & & & VEGF signaling pathway & 0.119 & 0.039 \\
\hline
\end{tabular}


Table 4. Solute carrier (SLC) family gene expression is significantly impacted by $6 \mathrm{~h}$ MEHP treatment in WEC. The mitochondrial transport family (SLC25) represents the most significantly altered family of transporters, crucial for the catabolic processes of the cell.

\begin{tabular}{|c|c|c|c|c|}
\hline \multirow{2}{*}{ SLC family } & \multirow{2}{*}{ 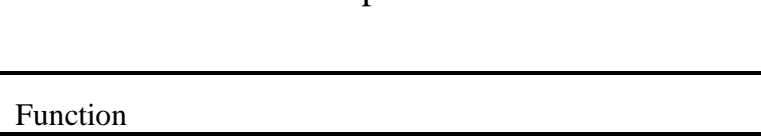 } & \multicolumn{3}{|c|}{ \# genes sig. changed } \\
\hline & & EMB & VYS & TOTAL \\
\hline 1 & glial high affinity glutamate/neutral amino acid transporter & 1 & 2 & 3 \\
\hline 4 & bicarbonate transporters & 6 & 2 & 8 \\
\hline 5 & inositol/sodium-dependent glucose transporters & 2 & 1 & 3 \\
\hline 6 & neurotransmitter transporters & 3 & 0 & 3 \\
\hline 7 & cationic/L-type amino acid transporters & 3 & 2 & 5 \\
\hline 8 & sodium/calcium exchangers & 1 & 1 & 2 \\
\hline 9 & sodium/hydrogen exchangers & 3 & 3 & 6 \\
\hline 10 & sodium/bile salt exchangers & 0 & 1 & 1 \\
\hline 11 & proton-coupled divalent metal ion transporters & 1 & 0 & 1 \\
\hline 12 & electroneutral cation-coupled chloride cotransporters & 2 & 1 & 3 \\
\hline 13 & sodium sulphate/carboxylate exchangers & 0 & 1 & 1 \\
\hline 16 & monocarboxylic acid transporters & 6 & 2 & 8 \\
\hline 17 & phosphate/organic anion transporters & 2 & 1 & 3 \\
\hline 19 & folate/thiamine transporters & 1 & 1 & 2 \\
\hline 21 & organic anion transporters & 4 & 3 & 7 \\
\hline 22 & organic cation/anion transporters & 4 & 0 & 4 \\
\hline 23 & ascorbate/nucleobase transporters & 1 & 1 & 2 \\
\hline 24 & sodium/potassium/calcium exchangers & 1 & 0 & 1 \\
\hline 25 & mitochondrial transporters & 9 & 6 & 15 \\
\hline 26 & sulfate/anion transporters & 2 & 2 & 4 \\
\hline 29 & nucleoside transporters & 1 & 0 & 1 \\
\hline 30 & zinc transporters & 2 & 2 & 4 \\
\hline 31 & copper transporters & 1 & 0 & 1 \\
\hline 33 & acetyl-CoA transporters & 0 & 1 & 1 \\
\hline 35 & nucleotide sugar transporters & 8 & 1 & 9 \\
\hline 36 & proton-coupled amino acid transporters & 0 & 1 & 1 \\
\hline 37 & sugar phosphate exchangers & 1 & 0 & 1 \\
\hline 38 & sodium-amino acid cotransporters & 1 & 0 & 1 \\
\hline 39 & zinc/metal ion transporters & 1 & 3 & 4 \\
\hline 44 & choline transporter-like family & 1 & 2 & 3 \\
\hline 45 & putative sugar transporters & 2 & 1 & 3 \\
\hline 46 & folate transporters & 1 & 1 & 2 \\
\hline
\end{tabular}




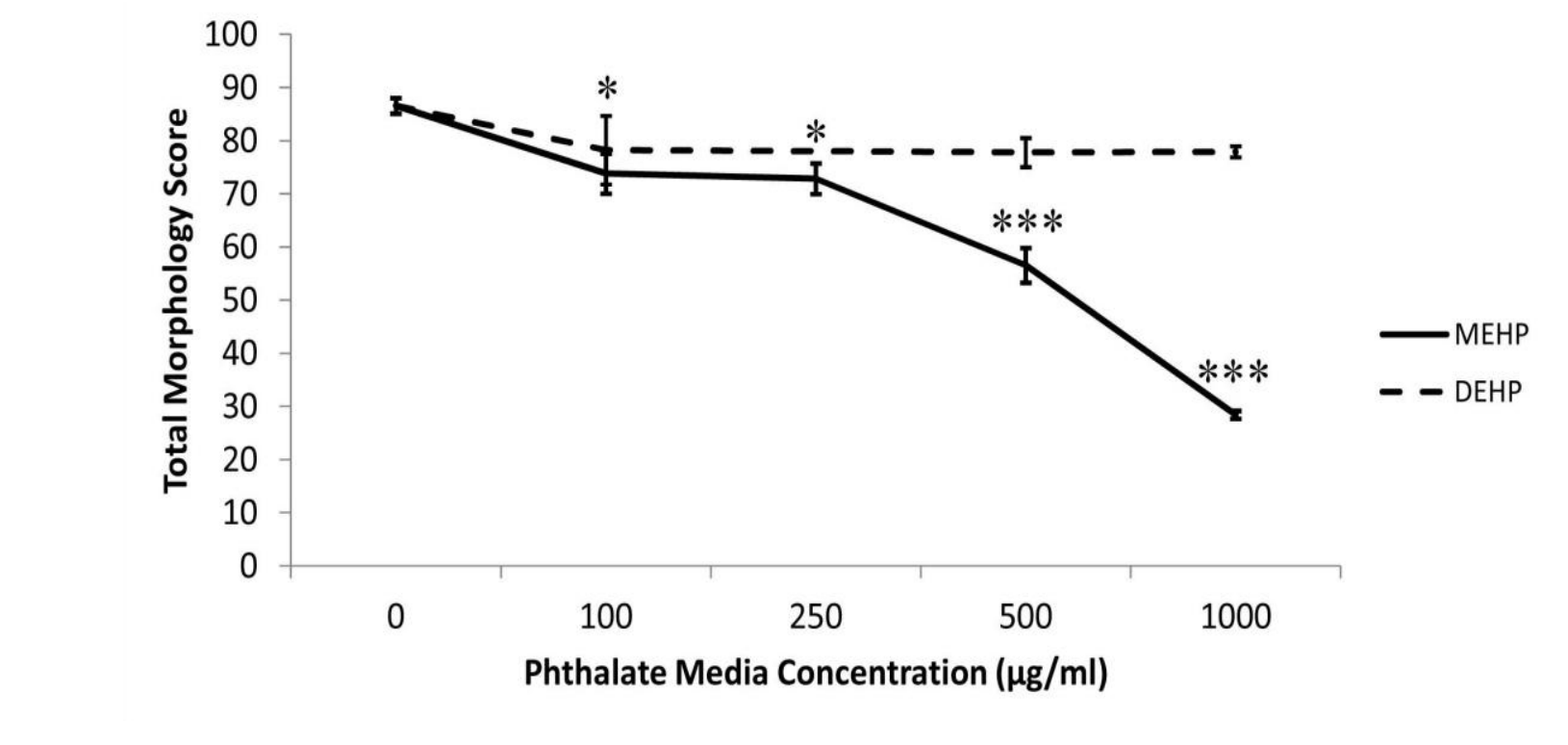

\section{Figure}

Phthalate Media Concentration $(\mu \mathrm{g} / \mathrm{ml})$

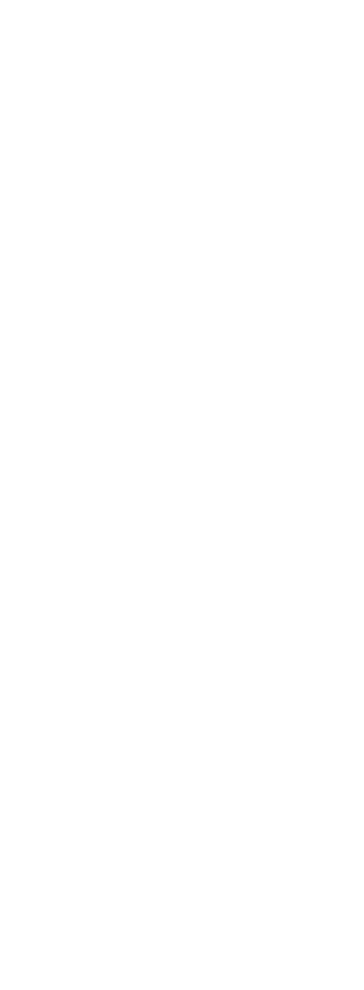

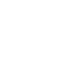

.

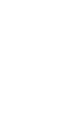

.

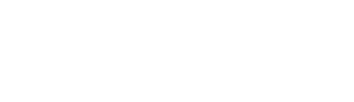



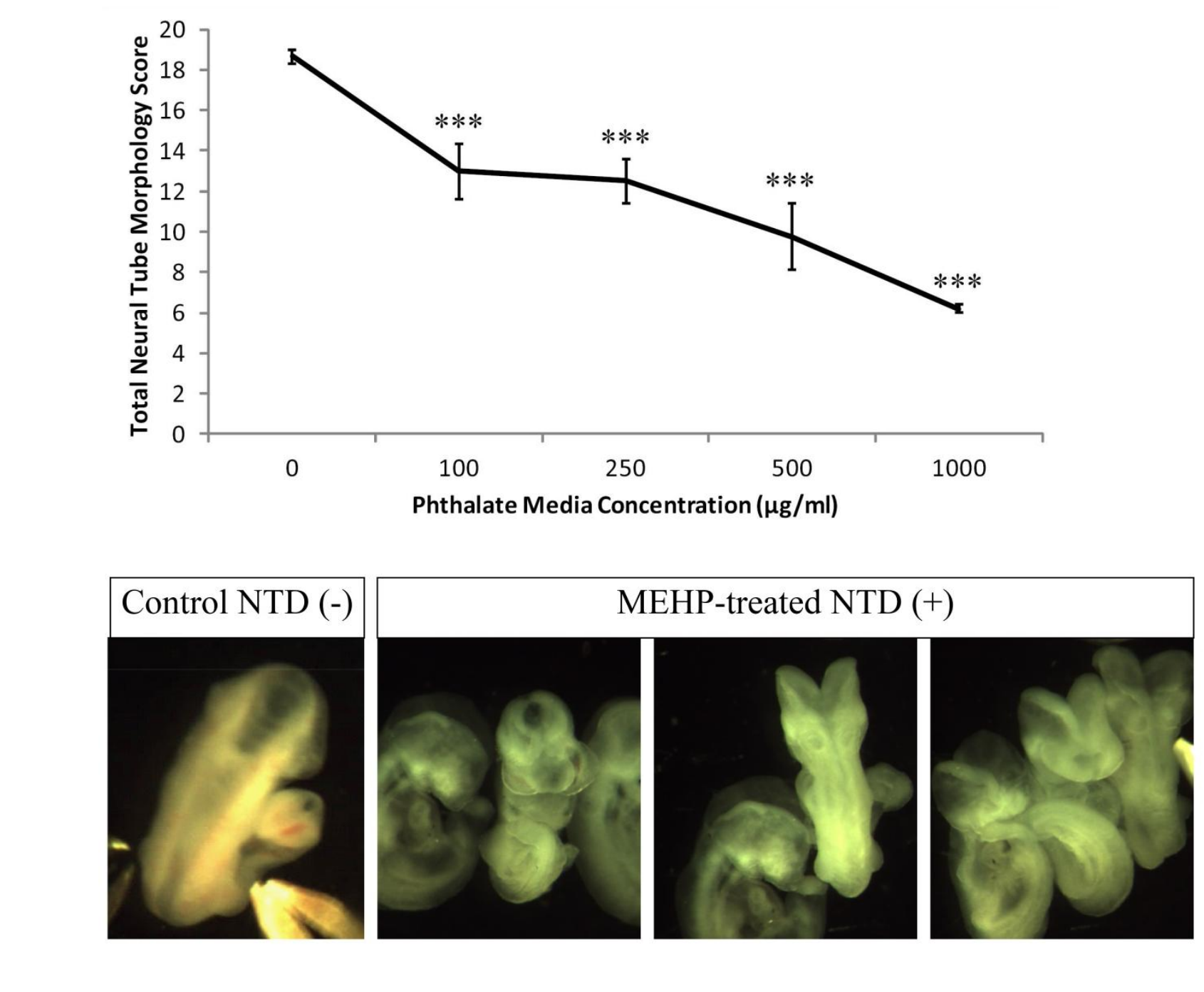

Phthalate Media Concentration ( $\mu \mathrm{g} / \mathrm{ml})$

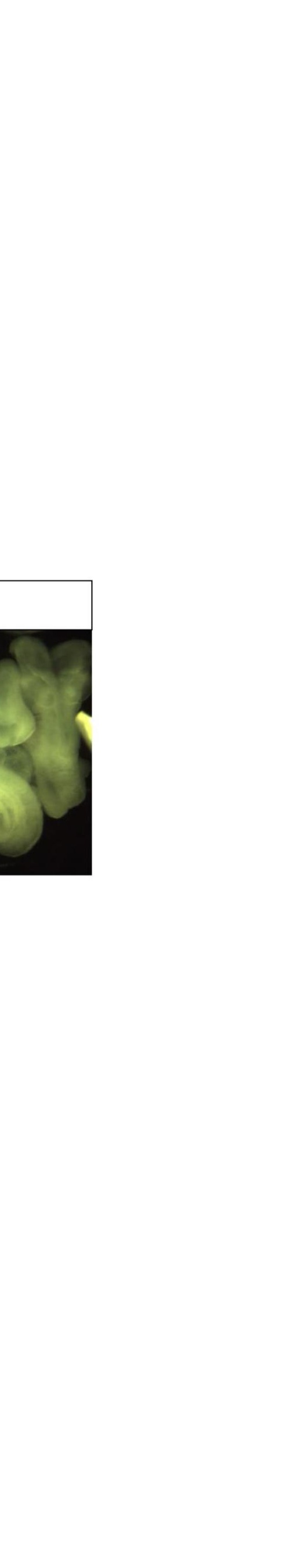


Figure 4

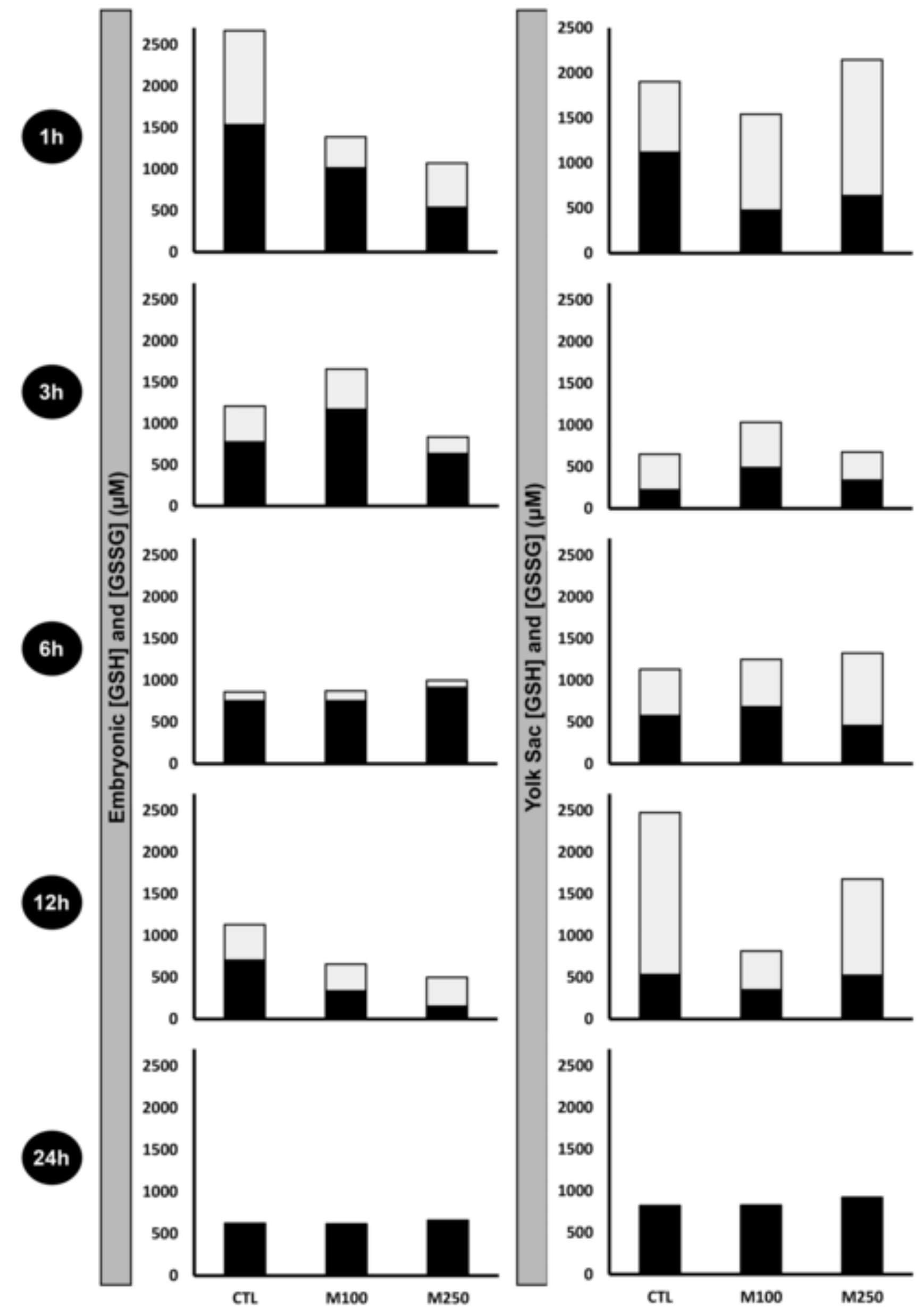


Figure 5

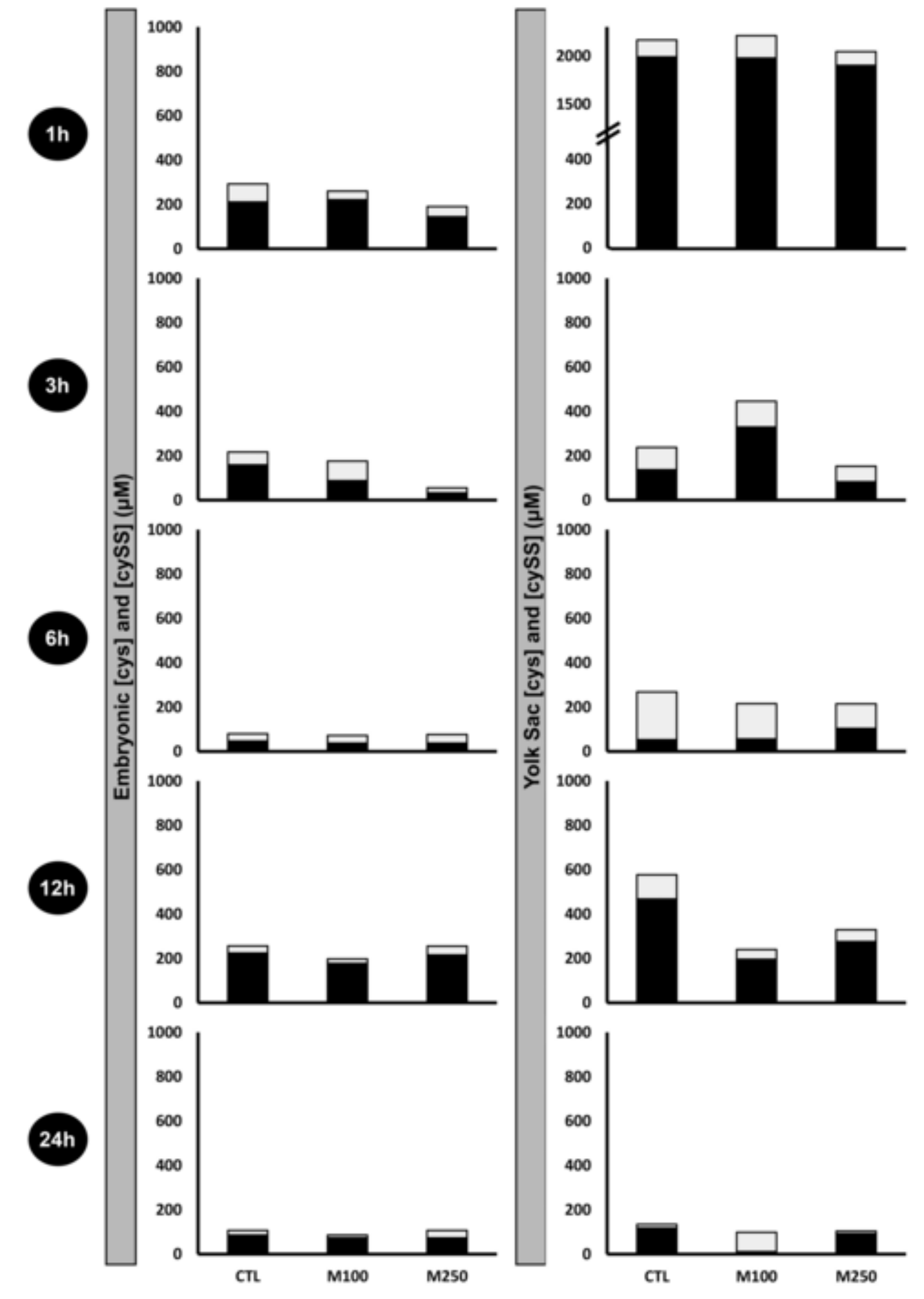


EMB
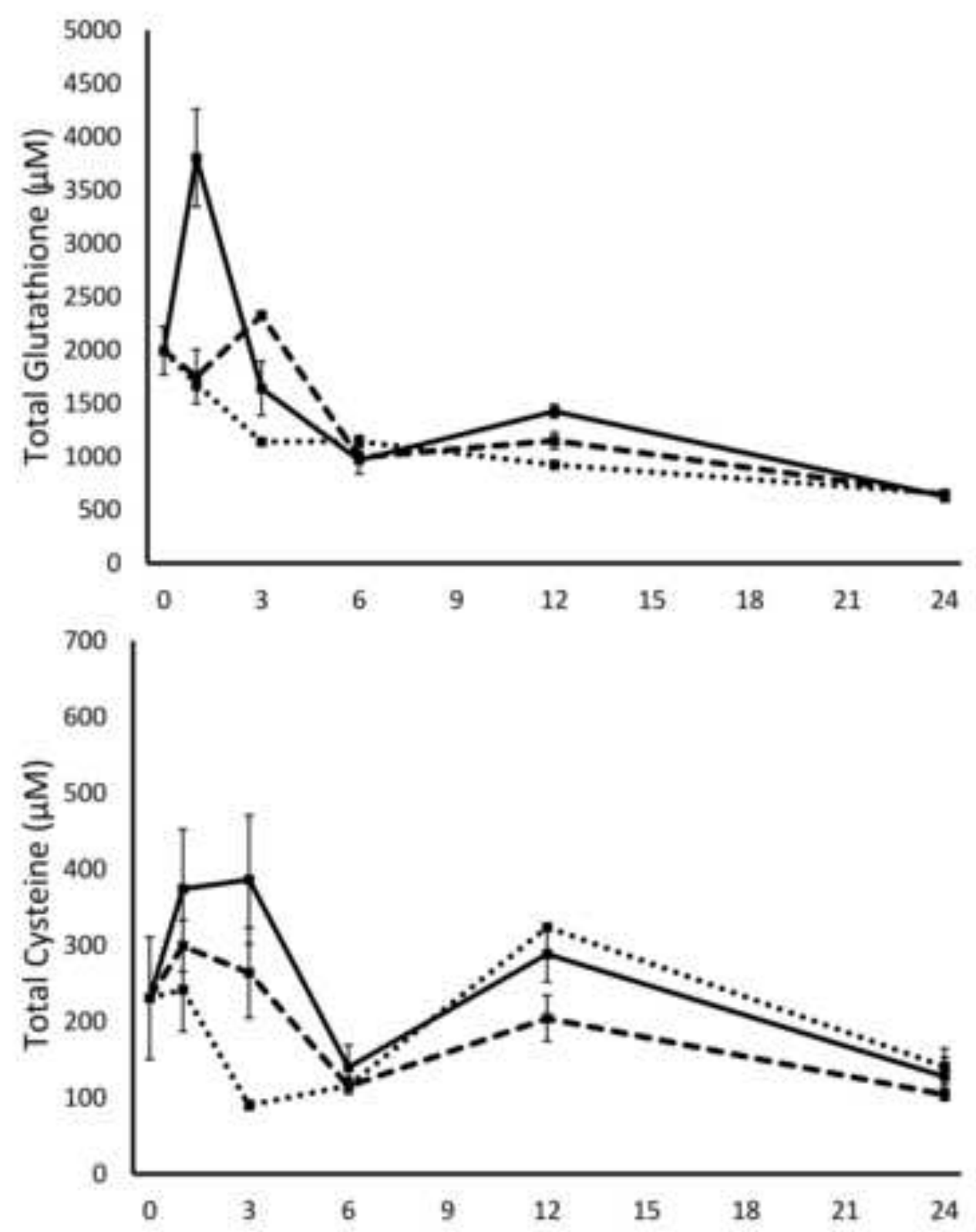

VYS
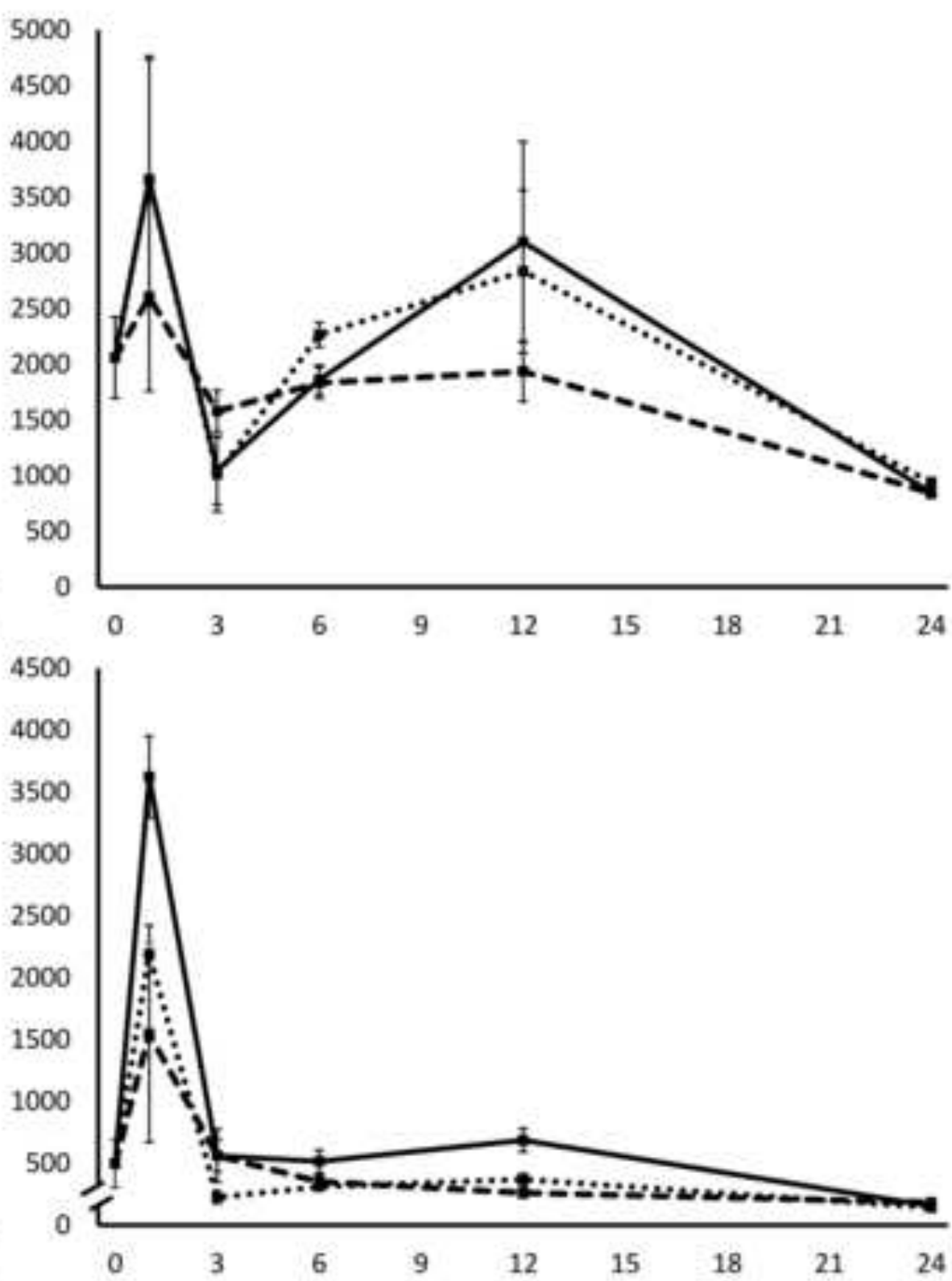

-Control (DMSO) $\quad$ - MEHP $(100 \mu \mathrm{g} / \mathrm{ml}) \quad \cdots \cdots$ MEHP $(250 \mu \mathrm{g} / \mathrm{ml})$ 


\section{EMB}
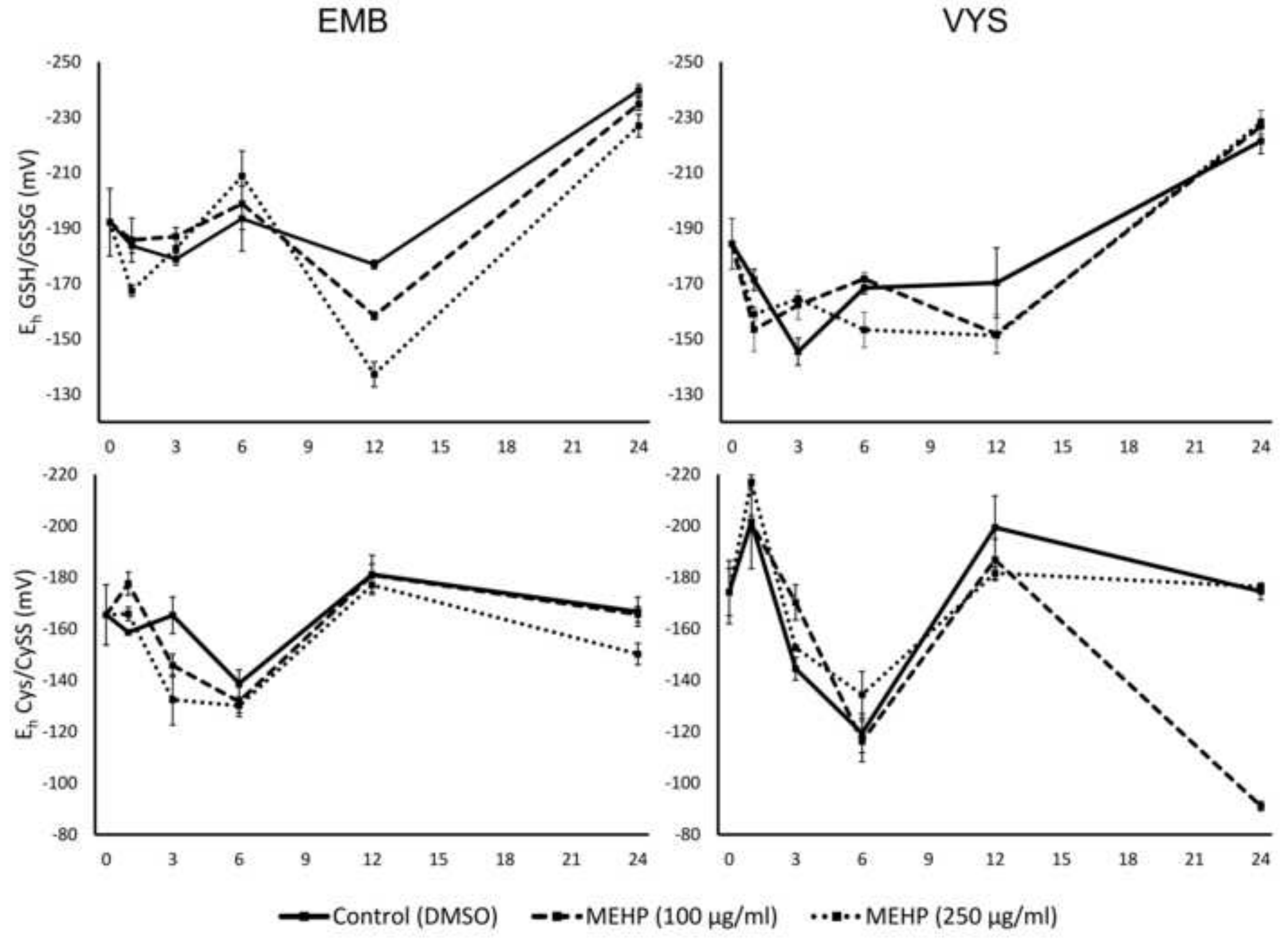

VYS
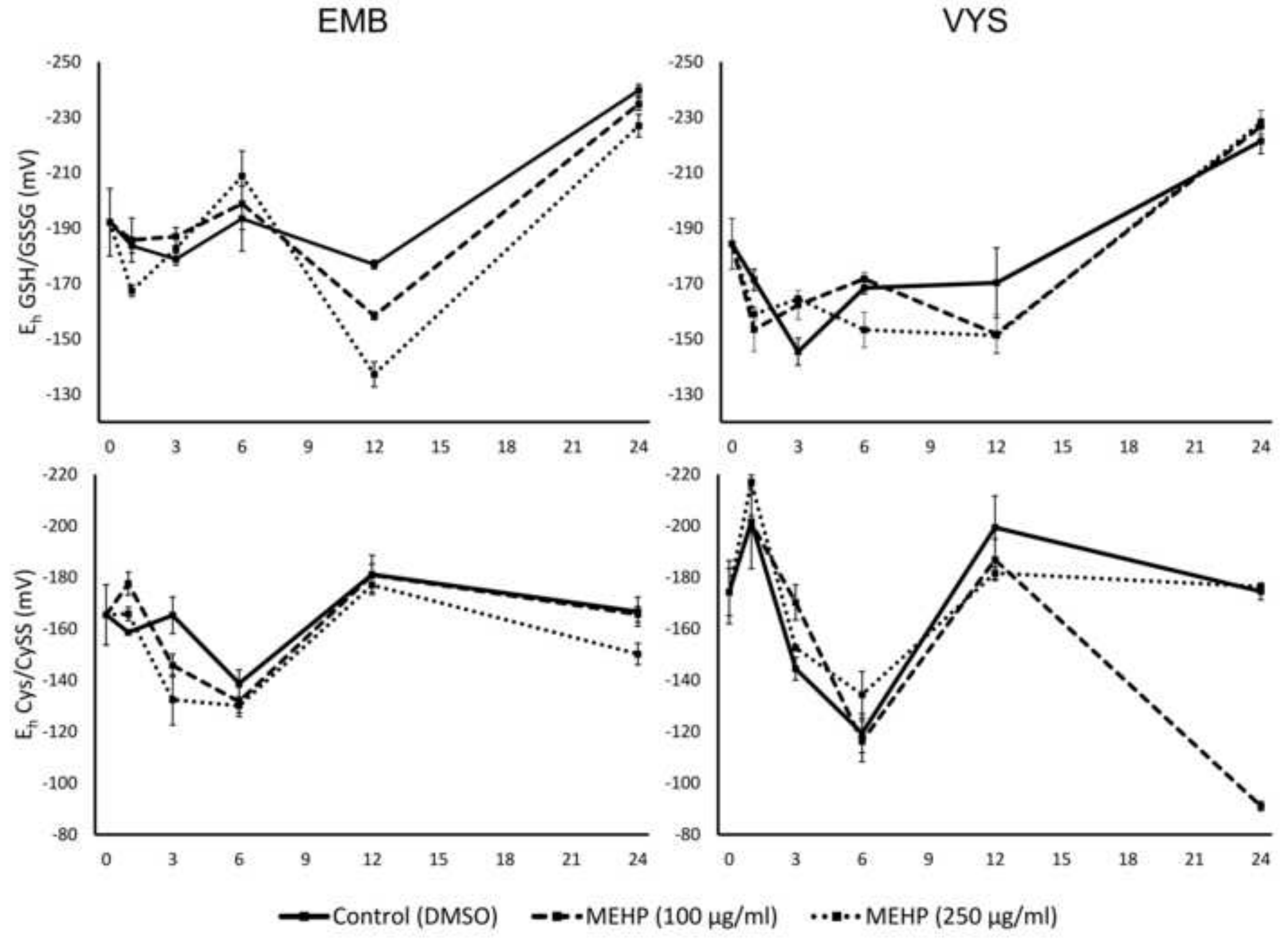

$\rightarrow$ Control (DMSO) -m-MEHP $(100 \mu \mathrm{g} / \mathrm{ml}) \quad \cdots \cdots$ MEHP $(250 \mu \mathrm{g} / \mathrm{ml})$ 


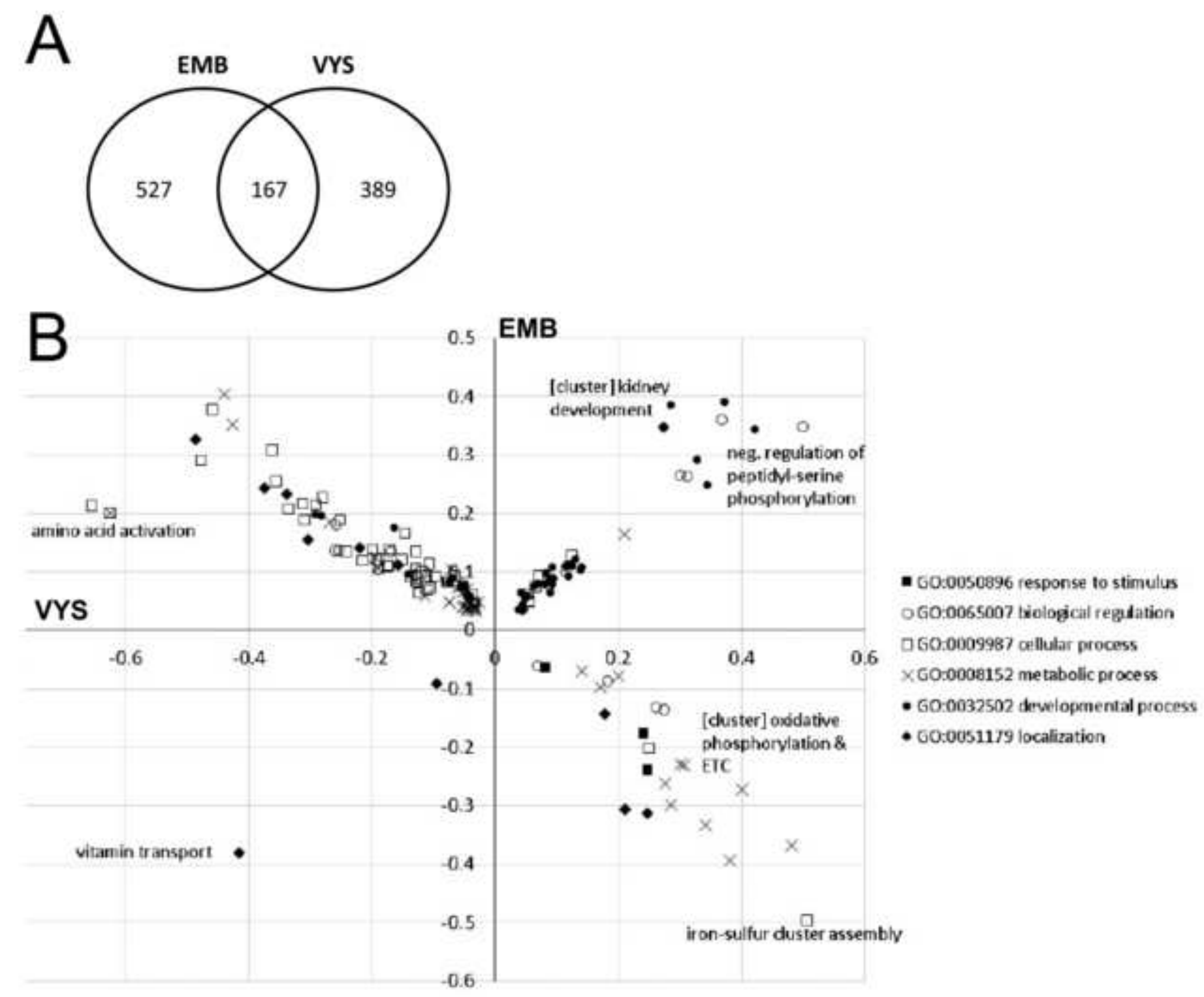

